\section{Como a Estratégia como Prática pode Habilitar a Inovação sob Influência do Dinamismo Ambiental?}

\author{
How can Strategy-as-Practice Enable Innovation under the Influence of \\ Environmental Dynamism?
}

\author{
Adilson Carlos Yoshikuni*1 1 \\ José Eduardo Ricciardi Favaretto ${ }^{20}$ \\ Alberto Luiz Albertin ${ }^{10}$ \\ Fernando de Souza Meirelles ${ }^{10}$
}

\section{RESUMO}

Objetivo: o objetivo deste estudo foi analisar a inovação em organizaçóes brasileiras quando habilitada por meio de sistemas de informação estratégicos (SIS), na abordagem de estratégia como prática, sob a influência da moderação do dinamismo. Métodos: foi utilizada a modelagem de equação estrutural pela técnica estatística de partial least squares path modeling (PLS-PM) e estudo pós-análise (post hoc analysis) para identificação da heterogeneidade não observada, com uma amostra de 256 empresas brasileiras de diferentes setores. Resultados: os resultados revelaram que o SIS apresentou influência na inovação de exploitation e exploration. A moderação do dinamismo não se apresentou na relaçáo entre o SIS e a inovaçáo de exploitation. Contudo, o SIS mostrou influência na inovação de exploration em altos níveis de dinamismo. A heterogeneidade não observada foi identificada para dois segmentos da amostra, demonstrando forte efeito de caminho entre o SIS e inovação de exploration e exploitation com a alta presença de dinamismo em empresas de serviços e de tamanho superior a 500 funcionários. Conclusóes: o estudo contribuiu para a extensão da literatura de estratégia como prática e inovação, ao identificar o SIS como alternativa para habilitar a inovação na organização, em ambientes com alta taxa de mudança e imprevisibilidade.

Palavras-chave: estratégia como prática; strategic information systems; inovação de exploration e exploitation; dinamismo ambiental; planejamento estratégico contemporâneo.

\footnotetext{
* Autor Correspondente.

1. Fundação Getulio Vargas, Escola de Administração de Empresas de São Paulo, Departamento de Tecnologia e Ciência de Dados, São Paulo, SP, Brasil.

2. Escola Superior de Propaganda e Marketing, Programa de Pós-graduação em Administração, São Paulo, SP, Brasil.

Como citar: Yoshikuni, A. C., Favaretto, J. E. R., Albertin, A. L., \& Meirelles, F. de S. (2022). Como a estratégia como prática pode habilitar a inovação sob influência do dinamismo ambiental? Revista de Administração
} Contemporânea, 26(1), e200131. https://doi.org/10.1590/1982-7849rac2022200131.por

\section{ABSTRACT}

Purpose: the objective of this study was to analyze innovation in Brazilian organizations when enabled through SIS, in the strategy-as-practice approach, on the influence of moderating environmental dynamism. Methods: we performed structural equation modeling using the statistical technique of partial least squares path modeling (PLS-PM) and post hoc analysis to identify unobserved heterogeneity, with a sample of 256 Brazilian companies from different sectors. Results: the results revealed that the SIS had a strong and significant influence on the innovation of exploitation and exploration. The environmental uncertainty of dynamism moderation was not present in the relationship between SIS and the innovation of exploitation. However, SIS showed a strong and significant influence on innovation of exploration in high levels of dynamism. The existence of unobserved heterogeneity was identified for two segments of the sample, demonstrating a strong path effect between SIS and innovation of exploration and exploitation on the high presence of dynamism in service companies with a size greater than 500 employees. Conclusions: the study contributed to the extension of the strategy-as-practice and innovation literature, by identifying SIS as an alternative in the planning process to enable innovation in the organization, in environments with a high rate of change and unpredictability.

Keywords: strategy-as-practice; strategic information systems; innovation of exploration and exploitation; environmental dynamism; contemporary strategic planning.

Classificação JEL: M150.

Editor-chefe: Wesley Mendes-da-Silva (Fundação Getulio Vargas, EAESP, Brasil) Pareceristas: Simone Vasconcelos Ribeiro Galina (Universidade de São Paulo, FEA-RP, Brasil) (f)

Relatório de Revisão por Pares: A disponibilização do Relatório de Revisão por Pares não foi autorizada pela revisora. Recebido: 09/05/2020 Última versão recebida em: 24/09/2020 Aceite em: 24/09/2020

\# de revisores convidados até a decisão:

\begin{tabular}{|c|c|c|c|c|c|c|c|c|c|}
\hline 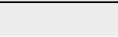 & 1 & 2 & 3 & 4 & 5 & 6 & 7 & 8 & 9 \\
\hline \multicolumn{10}{|l|}{$1^{a}$ rodada } \\
\hline $2^{a}$ rodada & $x$ & x & $\stackrel{0}{2}$ & (x) & & & & & \\
\hline $3^{a}$ rodada & 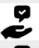 & & & & & & & & \\
\hline & $\stackrel{9}{6}$ & & & & & & & & \\
\hline
\end{tabular}




\section{INTRODUÇÃO}

Estudos sobre o processo de planejamento estratégico dos autores Song, Im, Van Der Bij e Song (2011) e Arend, Zhao, Song e Im (2017) demonstraram efeitos negativos nas atividades de inovação. Entretanto, a evolução do planejamento estratégico (Wolf \& Floyd, 2017) na abordagem do corpo teórico da estratégia como prática (Marabelli \& Galliers, 2017; Whittington, 2014) traz o papel da tecnologia como facilitadora na agilidade, flexibilidade e experimentação para o processo de planejamento estratégico. De acordo com Whittington (2014), a formulação, comunicação, execução e acompanhamento das estratégias nas organizaçóes são inseparáveis do suporte dos sistemas de informaçấo estratégicos ou strategic information systems (SIS).

Os estudos de inovação (exploration e exploitation) emergiram de perspectivas teóricas no campo da aprendizagem organizacional (Jansen, Van Den Bosch, \& Volberda, 2006; March, 1991), e posteriormente alcançaram outros campos como, por exemplo, estratégia como prática (Whittington, 2014; Wolf \& Floyd, 2017) e sistemas de informação (SI) (Marabelli \& Galliers, 2017; Merali, Papadopoulos, \& Nadkarni, 2012; Yoshikuni \& Albertin, 2018).

O dinamismo ambiental (Mikalef \& Pateli, 2017) é um fator externo que pressiona as organizaçôes a buscarem inovação (Chen, Zeng, Lin, \& Ma, 2017; Jansen et al., 2006; Jansen, Vera, \& Crossan, 2009), sendo crescente nos últimos anos (Kamasak, Yozgat, \& Yavuz, 2017; Panda \& Rath, 2018). As empresas pressionadas pela rápida mudança do ambiente de negócios (dinamismo ambiental) reforçam a necessidade de se tornarem inovadoras. Recente estudo a respeito do SIS na abordagem da estratégia como prática (Yoshikuni \& Albertin, 2018) demonstrou efeitos positivos ao promover a capacidade e flexibilidade para criar estratégias competitivas de inovaçáo em resposta a mudanças ambientais.

A literatura sugere pesquisas em novos contextos, circunstâncias e necessidades (Jarzabkowski \& Kaplan, 2015; Moeini, Rahrovani, \& Chan, 2019), em busca de relevância da estratégia na prática. Este trabalho buscou estudar tais fenômenos (estratégia como prática, SIS, inovação e dinamismo ambiental) no contexto de organizações brasileiras, onde não foram identificados estudos destas relaçóes.

A pergunta de pesquisa que este estudo se propôs a responder foi: Como a estratégia como prática por meio do SIS pode habilitar a inovaçáo de exploration e exploitation nas organizaçóes, sob influência do dinamismo ambiental? O objetivo deste estudo foi analisar a inovação em organizaçóes brasileiras quando habilitada por meio de SIS, na abordagem de estratégia como prática, sob a influência da moderação do dinamismo ambiental.

O estudo contribuiu para a extensão da literatura de estratégia como prática e inovação, considerando as lacunas de conhecimento (gaps) ainda existentes no campo (Marabelli \& Galliers, 2017; Whittington, 2014), ao identificar o SIS como alternativa no processo de planejamento para habilitar a inovação. Além disso, há carência de pesquisas, como mencionado em estudo conceitual por Wolf e Floyd (2017), sugerindo esforços para novos trabalhos empíricos com foco em ratificar estudos conceituais em planejamento estratégico contemporâneo, onde a estratégia como prática seria uma das abordagens. Estudos de pesquisadores do campo de sistemas de informaçáo (SI) (Marabelli \& Galliers, 2017; Whittington, 2014) também reforçaram a necessidade de realização de novas pesquisas relacionadas ao SIS nesta mesma abordagem.

\section{REVISÃO DA LITERATURA E HIPÓTESES}

\section{Inovação de exploration e exploitation}

Proposta por March (1991, 1995), a teoria da aprendizagem organizacional descreve duas maneiras distintas e complementares pelas quais as organizaçóes aprendem: exploration e exploitation. As iniciativas de exploration estão associadas com as atividades que aumentam a variação nos processos, funções e tarefas organizacionais, incluindo a invenção, o controle sem rigidez, a tomada de riscos e novas propostas de valores para os clientes, que compreendem ações organizacionais de busca, descoberta, experimentação e assunção de riscos (Gupta, Smith, \& Shalley, 2006; Popadiuk, 2012; Scandelari \& Cunha, 2013).

Assim, as atividades de exploration envolvem experimentação de novas ideias, paradigmas, tecnologias, estratégias e conhecimentos, com a intenção de descobrir alternativas que venham superar ou no mínimo atender as necessidades do mercado (Jansen et al., 2009; Lewin \& Volberda, 1999; Xue, Ray, \& Sambamurthy, 2012). As empresas que se posicionam com práticas de inovação de exploration desenvolvem capacidade para mapear frequentemente o ambiente externo geral com objetivo de identificar fatores que potencializam o lançamento de novos produtos e serviços, para então se diferenciarem dos competidores e se estabelecerem como empresas de vanguarda (Chen, Mocker, Preston, \& Teubner, 2010; Ho \& Lu, 2015; Mintzberg, Ahlstrand, \& Lampel, 2009).

As estratégias de exploitation relacionam-se à inovação por meio do aproveitamento dos recursos, processos e estratégias em inovaçóes incrementais (March, 1991; Scandelari \& Cunha, 2013), e são projetadas para 
atender as necessidades de clientes e mercados atuais (Ho \& Lu, 2015; Maletič, Maletič, \& Gomišček, 2016; Popadiuk et al., 2010). Assim, a essência da inovação de exploitation busca a melhoria contínua das competências, tecnologias e paradigmas existentes (Gupta et al., 2006; Jansen et al., 2006). Segundo Jansen, Van Den Bosch e Volberda (2006), a inovação de exploitation apoia-se em aperfeiçoar os produtos e serviços existentes, com pequenas e frequentes adaptaçóes no portfólio, para manter e/ou expandir a participação no mercado atual.

As empresas que adotam práticas de inovação de exploitation desenvolvem habilidades para aumentar a eficiência e a eficácia produtiva pela racionalização do uso dos recursos e pelo incremento de inovaçóes dos produtos e serviços existentes (Popadiuk, 2012). A inovaçấo de exploitation caracteriza-se pela aversão a riscos, buscando a melhoria contínua por meio das capacidades, competências e tecnologias existentes, na racionalização dos processos de negócios (Lewin \& Volberda, 1999; Popadiuk \& Bido, 2016), legitimando a padronização, a automatização da rotina com forte apelo à produtividade para gerar ganhos de economia de escala (Gupta et al., 2006; Xue et al., 2012).

Normalmente, as inovaçóes de exploration (ou inovaçóes radicais) são aquelas inovaçôes focadas no alcance de clientes ou mercados emergentes, solicitando novos conhecimentos organizacionais, em contraste com a inovação de exploitation (ou inovaçôes incrementais), que são aquelas inovaçôes projetadas para atender as necessidades dos clientes existentes, com base em conhecimento organizacional (Benner \& Tushman, 2003).

\section{Strategic information systems (SIS) e inovação}

Estudos acadêmicos seminais demonstraram que o SIS habilita a estratégia (Chan \& Huff, 1992; King, 1978), dando suporte ao processo e conteúdo da estratégia de negócio (Arvidsson, Holmström, \& Lyytinen, 2014; Chen et al., 2010; Newkirk \& Lederer, 2006; Singh, Watson, \& Watson, 2002) e contribuindo para a sobrevivência, sustentação e crescimento das organizaçóes (Chan, Sabherwal, \& Thatcher, 2006; Chen et al., 2014; Marabelli \& Galliers, 2017) em ambientes complexos (Merali et al., 2012) e dinâmicos (Neirotti \& Raguseo, 2017).

O termo SIS é tratado na literatura acadêmica de SI com diversas abordagens teóricas (Chen et al., 2010; Merali et al., 2012), sem ter uma forma única em sua definição (Martinez-Simarro, Devece, \& Llopis-Albert, 2015; Peppard, Galliers, \& Thorogood, 2014). O SIS pode ser visto como um conjunto de recursos de TI/SI, abrangendo coleta, armazenamento, processamento, análise e disponibilização de dados/informaçáo para apoiar a tomada de decisão e o suporte aos processos de gestấo da estratégia (Chan et al., 2006; Yoshikuni \& Albertin, 2018).

A definição do SIS utilizada neste artigo é baseada no recente estudo de Yoshikuni e Albertin (2018), destacada por Kaplan e Norton (2008), como os recursos de TI/SI incorporados ao processo de planejamento estratégico nas fases de conscientização estratégica, análise de situação, concepçáo de estratégia, formulação, implementação e acompanhamento da estratégia de negócio. Outros estudos sobre SIS investigaram sua influência em habilitar as iniciativas de inovaçáo (Chan et al., 2006; Chen et al., 2010; Johnson \& Lederer, 2013; Leidner, Lo, \& Preston, 2011; Sabherwal \& Chan, 2001) sob a influência do dinamismo ambiental externo (Merali et al., 2012; Mikalef \& Pateli, 2017).

A literatura sobre SIS (Merali et al., 2012; Nan \& Tanriverdi, 2017; Pavlou \& El Sawy, 2010) reitera a necessidade do direcionamento de pesquisas para lidar com o aumento da turbulência, incerteza e dinamismo no cenário competitivo. Embora a importância do SIS na inovação tenha sido frequentemente destacada (Chen et al., 2010; Chuang \& Lin, 2017; Leidner et al., 2011; Sabherwal \& Chan, 2001), são evidenciadas lacunas sugerindo estudos com abordagem de inovação de exploration e exploitation habilitada pelo SIS com a influência do dinamismo ambiental (Pavlou \& El Sawy, 2010; Schilke, 2014).

O framework conceitual proposto por Galliers (2011) evidenciou aspectos teóricos para a compreensão do interrelacionamento do SIS com a inovação de exploration e exploitation, que visa a apoiar processos de comunicação e colaboração e alavancar conhecimento pela aprendizagem organizacional relacionada à estratégia sob a influência do dinamismo do ambiente. Contudo, não foram identificados estudos posteriores (sobre o SIS) que analisaram tal relação e seus efeitos com uso do framework conceitual proposto por Galliers (2011). Em estudo recente de SI (Teubner, 2013), foi destacado que as organizaçóes precisam reagir às mudanças impostas pela incerteza do ambiente de forma orquestrada e organizada, apresentando o SIS como um instrumento de gerenciamento estratégico de conhecimento e aprendizado organizacional.

Estudos adicionais demonstram que o SIS habilita as competências essências para a organização desenvolver efetivamente iniciativas de criatividade e/ou de produtividade/controle (Chen et al., 2010; Merali et al., 2012). A inovação de exploration foca na criatividade da empresa pela geraçáo de novos produtos e serviços e novas abordagens apoiadas pelo SIS (Leidner et al., 2011), enquanto a inovação de exploitation é habilitada pelo SIS para desenvolver capacidades com foco no controle, ou seja, para a eficiência e produtividade organizacional (Philip, 2007; Xue et al., 2012). 
De acordo com Teubner (2013), o SIS contribui para a inovaçáo de exploration ao habilitar os processos informais e criativos de estratégias na organização. Estes são, acima de tudo, processos de coordenação e aprendizagem que ocorrem em equipes de planejamento individuais em vários níveis da organização, desde o alto gerenciamento até os comitês do projeto. Assim, o SIS habilita a comunicação, integraçáo e cooperação das iniciativas estratégicas 'de cima para baixo' (top-down) e 'de baixo para cima' (bottom-up), dando apoio aos acordos dos objetivos-alvo nos diversos níveis da empresa (Chen et al., 2010; Kaplan \& Norton, 2008).

As atividades de inovaçáo de exploration são alimentadas pelas informaçóes capturadas no ambiente externo (Jansen et al., 2006). Por meio das tecnologias digitais, o SIS identifica, coleta, processa e analisa um amplo volume de dados e possibilita à empresa desenvolver estratégias de vanguarda (Davenport, Harris, \& Morison, 2010; Jarzabkowski \& Kaplan, 2015; Yoshikuni \& Albertin, 2018). Assim, o SIS dá suporte às iniciativas de exploration ao disponibilizar informação para análise do ciclo de vida dos produtos e serviços, simulando a natureza absoluta do porfólio e a curva de ruptura do produto/serviço (Merali et al., 2012).

A inovação de exploitation é caracterizada por análises deliberadas, orientadas para o objetivo e parcialmente formalizadas em processos de decisão (Teubner, 2013). As iniciativas de exploitation são suportadas por aplicaçôes de TI/SI e objetivos operacionais predeterminados (Philip, 2007). Ou seja, as iniciativas de exploitation concentramse em executar com eficiência e eficácia atividades que contribuem para a produtividade organizacional por meio de inovaçóes incrementais (Jansen et al., 2006). Assim, as atividades de controle e acompanhamento são habilitadas pelo SIS e contribuem para as iniciativas de exploitation (Merali et al., 2012; Yoshikuni \& Albertin, 2018), por meio de aplicaçóes de sistema de gestáo de desempenho que consolidam e integram dados e informaçóes para mensurar a efetividade das atividades planejadas versus realizadas (Kaplan \& Norton, 2008).

Dessa forma, o SIS habilita a efetividade da estratégia de negócio para criar valor e benefícios para o processo e conteúdo das estratégias de inovação de exploitation - ao desenvolver estratégias para defender o mercado conquistado, com base na eficiência operacional e melhorias incrementais nos produtos/serviços (Philip, 2007; Yoshikuni \& Albertin, 2018), e com a inovação de exploration - ao habilitar a organização para compreender e atender as mudanças de mercado (Wilden \& Gudergan, 2014) e desenvolver estratégias de proatividade (prospecção), apoiando as decisões estratégicas de forma ágil e eficaz (Chan et al., 2006; Sabherwal \& Chan, 2001; Xue et al., 2012).
O SIS habilita a flexibilidade e agilidade durante a formulação do planejamento estratégico e a implantação de estratégias de negócios (Kearns \& Sabherwal, 2006; Yoshikuni \& Albertin, 2018) e inovação de exploration e exploitation (Johnson \& Lederer, 2013; Marabelli \& Galliers, 2017; Merali et al., 2012). Sob a lente de Chan e Huff (1992) e Johnson e Lederer (2013), o SIS capacita os diversos tipos de posturas estratégicas, tais como: de agressividade, análise, defesa interna, defesa externa, planejamento futurista, proatividade, tomada de risco e inovação. As diversas posturas estratégicas descritas (Chan et al., 2006) refletem as açôes de inovação de exploration e exploitation.

Dessa forma, é prevista a hipótese de que o SIS (incorporado ao processo da estratégia de negócio) influencia a inovação de exploration e exploitation na organização.

H1a: O SIS influencia positivamente a inovação de exploration.

H1b: O SIS influencia positivamente a inovação de exploitation.

\section{Dinamismo ambiental, strategic information systems e inovação}

O dinamismo ambiental tem sido estudado amplamente como fator que desafia as organizaçóes a responderem com agilidade e flexibilidade às necessidades do ambiente externo (Barbero, Ramos, \& Chiang, 2017; Chen et al., 2017; Schilke, 2014). Pesquisadores de SI também destacam fatores contextuais que influenciam a relação do SIS com a efetividade organizacional (Merali et al., 2012; Newkirk \& Lederer, 2010; Ray, Wu, \& Konana, 2009; Sohn, You, Lee, \& Lee, 2003; Yayla \& Hu, 2012). A amplitude e as mudanças impostas pela competição, tecnologias, novos hábitos dos consumidores, volatilidade e instabilidade no ambiente externo são dimensóes que caracterizam o dinamismo ambiental (Jansen et al., 2006; Kamasak et al., 2017; Wilhelm, Schlömer, \& Maurer, 2015).

O dinamismo é definido pelo nível de turbulência ou instabilidade enfrentada em um ambiente que proporciona evidências substanciais de seus efeitos sobre o desempenho da organização (Barbero et al., 2017). O dinamismo ambiental é descrito pela taxa de mudança e a imprevisibilidade da mudança no ambiente externo de uma empresa, e é caracterizado por mudanças em tecnologias, variaçóes nas preferências do cliente e flutuaçóes na demanda de produtos ou fornecimento de materiais (Jansen et al., 2006, 2009).

O dinamismo, como fator da volatilidade externa, pressiona a organização para obter informaçóes de forma mais rápida, para entáo compreender e tomar decisóes 
neste ambiente em constante transformação (Chen et al., 2014; Mao, Liu, \& Zhang, 2014). A tecnologia habilita a organização a gerar novos conhecimentos e identificar novas oportunidades por meio da captura de informaçóes de mercado (Dameron, Lê, \& Lebaron, 2015; George, Haas, \& Pentland, 2014; Yoshikuni \& Albertin, 2017), analisando amplo volume de dados (Rouhani, Ashrafi, Ravasan, \& Afshari, 2016; Shollo \& Galliers, 2016), transferindo dados de clientes e concorrentes, bem como gerando rápida disponibilidade de informação (Chen et al., 2014) para a tomada de decisão ágil e flexível (Pavlou \& El Sawy, 2006, 2010) e para formular e implantar iniciativas de exploration e exploitation (Mikalef \& Pateli, 2017).

A partir de evidências da presença de diferentes níveis de dinamismo (alto e baixo) (Chen et al., 2017), que refletem em diferentes efeitos de moderação sobre as iniciativas de inovação (Jansen et al., 2009) e exigem maior capacidade organizacional para enfrentar os desafios da incerteza ambiental (Chen et al., 2014; Mao et al., 2014), e com o apoio de estudos da literatura que relacionam modelos de alinhamento estratégico da tecnologia, demonstrando efeitos sobre a presença (alta e baixa) do dinamismo ambiental (Mikalef \& Pateli, 2017; Newkirk \& Lederer, 2006, 2010; Yayla \& Hu, 2012), foram formuladas duas hipóteses adicionais para o estudo.
H2a: A influência positiva entre o SIS e a inovação de exploration será mais forte quando o dinamismo ambiental for alto.

H2b: A influência positiva entre o SIS e a inovação de exploitation será mais forte quando o dinamismo ambiental for alto.

A tomada de decisão da organização frente à alta presença do dinamismo pode ser sustentada pelo SIS com o direcionamento prescritivo, ou seja, 'de cima para baixo', com maior ênfase nas iniciativas de exploitation por exemplo, via comitês diretivos de projetos de inovação (Arend, Zhao, Song, \& Im, 2017), e iniciativas emergentes que se caracterizam pela coordenação e aprendizagem de equipes e indivíduos em vários níveis da organização, pelo processo 'de baixo para cima' (Teubner, 2013).

\section{MÉTODO DE PESQUISA}

O instrumento de pesquisa foi desenvolvido com base no modelo ilustrado pela Figura 1, para mensurar variáveis de acordo com a operacionalizaçáo proposta e permitir testar as hipóteses da pesquisa.

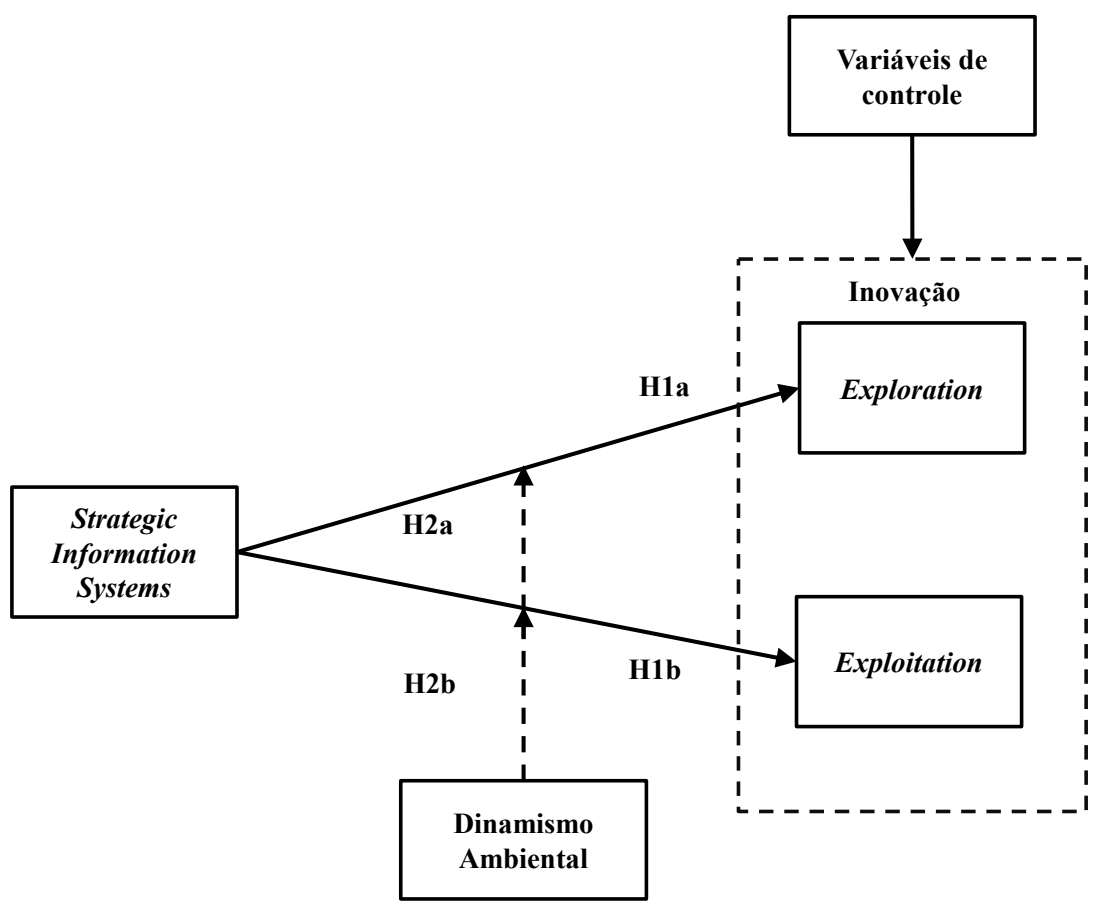

Figura 1. Modelo conceitual da pesquisa.

As variáveis de controle usadas no modelo foram o tamanho da empresa e o setor. Fonte: Elaborado pelos autores. 
Nesse modelo, foram utilizadas variáveis discutidas na literatura acadêmica, como descrito na Tabela 1, incluindo a variável de marcador latente medido ou measured latent marker variable (MLMV), criada pelos autores para controlar (verificar) o viés do método comum de coleta.

$\mathrm{O}$ estudo mensurou o construto de inovaçáo de exploration e exploitation pelas medidas e itens no nível de unidade organizacional de Jansen et al. (2006). A mensuração do SIS foi obtida de estudos seminais disponíveis na literatura acadêmica de SI (Newkirk \& Lederer, 2006; Singh et al., 2002), com a utilização da escala já operacionalizada em recente estudo de Yoshikuni e Albertin (2018). Para a mensuração da incerteza do dinamismo ambiental foi utilizada a escala de Yayla e Hu (2012).

Tabela 1. Variáveis e sua forma de mensuração identificada na literatura acadêmica.

\begin{tabular}{lll}
\hline Variáveis do modelo & Mensuração & Referências \\
\hline Strategic information systems & $\begin{array}{l}\text { SI incorporado no processo de planejamento } \\
\text { estratégico }\end{array}$ & $\begin{array}{l}\text { Newkirk \& Lederer (2006); Singh, Watson, e Watson } \\
\text { (2002); Yoshikuni e Albertin (2018); }\end{array}$ \\
$\begin{array}{lll}\text { Inovação (exploration e exploitation) } & \text { Atividades de exploration e exploitation } & \text { Jansen et al. (2006) } \\
\text { Dinamismo ambiental } & \text { Dinamismo ambiental } & \text { Yayla e Hu (2012) } \\
\text { Variáveis de controle } & \text { Tamanho da empresa e setor } & \text { Benitez, Castillo, Llorens, e Braojos (2018); } \\
\text { Benitez, Llorens, e Braojos (2018) }\end{array}$ \\
\hline Variável MLMV & $\begin{array}{l}\text { Quatro indicadores sugeridos pelos autores para a } \\
\text { remoçáo do viés do método comum de coleta }\end{array}$ & Chin, Thatcher, Wright, e Steel (2013) \\
\hline
\end{tabular}

Nota. Fonte: elaborado pelos autores.

Procedeu-se a validação da escala por meio da análise de conteúdo por professores e pesquisadores especialistas da área de TI/SI com mais de 10 anos de experiência em docência, pesquisas acadêmicas (com publicaçóes em periódicos e congressos da área) e consultoria de TI/SI, conforme recomendado por Morgado, Meireles, Neves, Amaral e Ferreira (2018).

Como sugerido por Wieland, Durach, Kembro e Treiblmaier (2017), foram seguidos procedimentos para garantir o rigor, a confiabilidade, a validade e a parcimônia nos itens da escala. $\mathrm{O}$ instrumento de coleta (questionário) continha as assertivas com escala de concordância tipo Likert de sete pontos, variando de um (discordo totalmente) a sete (concordo totalmente) - disponível no Apêndice A. Todos os indicadores das escalas foram validados pelos especialistas já citados, os quais realizaram ajustes semânticos com objetivo de torná-los ainda mais compreensíveis, sem afetar a validação de conteúdo.

Com o feedback obtido dos especialistas, ficou também esclarecido que a avaliação dos indicadores apontava a adequação dos construtos. Conforme recomendado por Hair, Hult, Ringle e Sarstedt (2017), as variáveis latentes do modelo proposto tiveram no mínimo três indicadores, permitindo a mensuração adequada dos construtos.

De acordo com a recomendação de Schwarz, Rizzuto, Wolverton e Roldán (2017) para pesquisas no campo de sistemas de informação, o estudo incluiu procedimentos preventivos, destinados a minimizar o viés do método de coleta, tais como: escolha de respondentes qualificados para responder ao questionário, itens construídos em linguagem clara e concisa, contrabalanceamento da ordem das questóes e o anonimato do respondente, além do emprego de técnicas estatísticas para detectar e controlar os efeitos do viés durante a análise de dados, conforme sugerido por Mackenzie e Podsakoff (2012) e Fuller, Simmering, Atinc, Atinc e Babin (2016).

Recomendada como uma solução operativa adequada para lidar com o viés de coleta para modelos de PLS (Chin, Thatcher, Wright, \& Steel, 2013; LealRodríguez, Ariza-Montes, \& Morales-fernández, 2017), a variável de marcador latente medido ou measured latent marker variable (MLMV) foi utilizada. A variável marcadora selecionada náo deve pertencer ao mesmo domínio dos construtos que moldam o modelo de pesquisa, e deve ser obtida a partir de uma unidade de análise diferente (Wang $\&$ Hajli, 2017). Assim, para atender a técnica de MLMV, os autores criaram quatro indicadores formativos (Chin et al., 2013), informados na Figura 2, para ter a menor correlaçáo lógica possível com os outros construtos que faziam parte do modelo. Este estudo usou o procedimento de correção de nível de item (ILC) descrito em Chin et al. (2013). Para confirmar a relação de independência entre os indicadores, verificou-se a correlação de Pearson entre os indicadores MLMV, que demonstrou dois coeficientes estatisticamente significantes $(0,19 ; 0,13$; valor- $p<0,05)$, com correlaçáo positiva muito baixa (Bisquerra et al., 2004). 
MLMV_1: É fácil alcançar meus objetivos.

MLMV_2: Terei o meu próprio negócio.

MLMV_3: Tenho uma atitude positiva em relação aos outros.

MLMV_4: Eu sempre imagino minha casa no futuro.

Figura 2. Indicadores formativos utilizados para a análise de viés do modelo estrutural.

Os quatro indicadores que mensuraram a variável MLMV (measured latent marker variable, ou variável de marcador latente medido) foram informados seguindo a ordem de MLMV_1 a MLMV_4. Fonte: elaborado pelos autores.

Os resultados mostraram que a inclusão dos quatro indicadores MLMV (Chin et al., 2013) não apresentou mudanças significativas nos coeficientes de caminho e coeficientes de explicação $\left(R^{2}\right)$ das relaçóes entre SIS e inovação de exploration e entre SIS e inovação de exploitation. Os valores de ajuste do modelo são consistentes com as estimativas originais (Tabela 4) e sugerem que o viés do método de coleta não é um problema no estudo.

\section{Coleta de dados e perfil da amostra}

Os dados para testar as hipóteses de pesquisa foram coletados de empresas brasileiras por meio de um instrumento de pesquisa. Tais empresas foram selecionadas do diretório fornecido pelo Centro de Tecnologia de Informação Aplicada (FGVcia) da Fundação Getúlio Vargas (FGV), devido ao fato de seus respondentes serem gestores e executivos que representam organizaçóes de diversos setores e tamanhos, com conhecimento das práticas de gestão do negócio e da tecnologia, permitindo assim examinar as hipóteses do estudo. Além disso, os respondentes tinham conhecimento do propósito do FGVcia, o qual busca estimular e coordenar esforços de pesquisa sobre a tecnologia de informação para contribuir de forma sinérgica na geração de conhecimento acadêmico e empresarial.

Foram enviados 1.353 convites via e-mail às organizaçóes, e a coleta dos dados foi realizada por intermédio de um formulário on-line (survey) disponibilizado na internet. Levou-se em consideração para escolha dos respondentes a sua posiçáo/cargo, experiência e conhecimento a respeito do conteúdo dos construtos do modelo. A amostra de respondentes obtida foi de 256 organizaçóes (19\% dos convites enviados), podendo ser considerada uma amostra por conveniência (Etikan, Musa, \& Alkassim, 2016), ou seja, uma amostra não probabilística ou aleatória, que contém organizaçóes de uma populaçãoalvo com facilidade de acesso pelos pesquisadores. Diversos outros estudos no campo de SI (Chen et al., 2014; Gupta et al., 2006; Mikalef \& Pateli, 2017) também utilizaram amostras de conveniência para a validação empírica.
Não foram identificados dados faltantes (missing values) e nem erros de preenchimento do questionário, visto que a plataforma via internet foi configurada para restringir tais casos. Analisou-se a existência de outliers pela distância quadrada de Mahalanobis $\left(\mathrm{DM}^{2}\right)$ (Cousineau \& Chartier, 2017; Marôco, 2010) por meio do software SPSS, e a amostra não apresentou valores de $\mathrm{DM}^{2}$ elevados (residual estatístico máximo da variável de $\mathrm{DM}^{2}=11,756$, com valor de probabilidade de $\mathrm{p}<0,001)$, indicando a não existência de outliers multivariados.

A unidade de análise da pesquisa foi a organização, e a composição dos respondentes (executivos de negócios e de TI/SI) foi: $39 \%$ com cargos de superintendentes, presidentes ou diretores, $36 \%$ de gerentes e coordenadores e $25 \%$ de supervisores com poder de decisão. A Tabela 2 descreve a composição das organizaçóes da amostra conforme o setor de atuação e o número de funcionários. Ficou evidenciado que a amostra foi essencialmente formada por organizaçóes dos setores de serviços e indústria $(93 \%$ das empresas pesquisadas), e $40 \%$ das organizaçóes possuíam o número de 500 ou mais funcionários.

Tabela 2. Dados demográficos da amostra - setor e número de funcionários.

\begin{tabular}{lclc}
\hline & \multicolumn{2}{c}{ Setor } & \multicolumn{2}{c}{ Número de funcionários } \\
\hline Agronegócio & $4 \%$ & $\leq 9$ & $9 \%$ \\
Governo & $3 \%$ & $10-49$ & $11 \%$ \\
Indústria & $36 \%$ & $50-99$ & $16 \%$ \\
Serviço & $57 \%$ & $100-249$ & $14 \%$ \\
& & $250-499$ & $9 \%$ \\
& & $\geq 500$ & $40 \%$ \\
\hline
\end{tabular}

Nota. Fonte: Elaborado pelos autores.

\section{RESULTADOS EMPÍRICOS}

\section{Técnica estatística}

As hipóteses foram testadas usando o método partial least squares path modeling (PLS-PM), por meio do software 
SmartPLS v3 (Hair, Hult, Ringle, \& Sarstedt, 2017), que demonstrou ser adequado no teste das relaçóes entre variáveis latentes, na análise do viés de coleta por meio de indicadores formativos da variável MLMV, na análise da moderação de variável contínua, na comparação da diferença de efeitos de caminho em grupos diferentes da mesma amostra (PLSMGA) e na identificação da heterogeneidade não observada por meio das técnicas de PLS-FIMIX e PLS-POS.

$\mathrm{Na}$ área das Ciências Sociais em geral, o teste estatístico do 'power' deve resultar > 0,8 (Cohen, 1988), significando que há pelo menos $80 \%$ de chance de encontrar relacionamentos que existam (Goodhue, Lewis, \& Thompson, 2007). Os pesquisadores Peng e Lai sugerem que "uma análise post hoc do 'power' deveria ser conduzida para verificar a adequação ao estudo" (Peng \& Lai, 2012, p. 47). Seguindo neste caminho, Aguirre-Urreta e Rönkkö (2015) recomendaram que as práticas metodológicas para a avaliação do 'power' em estudos que utilizam PLS poderiam ser melhoradas com a inclusão de um relatório de análise do 'power'. Este estudo utilizou a prática de Aguirre-Urreta e Rönkkö (2015), encontrando resultados consistentes para a amostra de 256 casos e 'power' > 0,8 no teste das relações do modelo.

\section{Modelo de mensuração}

As variáveis latentes reflexivas foram sujeitadas aos testes de confiabilidade, validade convergente e validade discriminante. Os coeficientes estimados (outer loading) dos itens apresentaram significância estatística (valor-p < 0,001) e informaram valores acima de 0,7 , e no caso dos itens com os valores entre 0,4 e 0,7 foram mantidos apenas aqueles que aumentaram a confiabilidade composta $(\mathrm{CR})$ e a variância média extraída (AVE), conforme recomendado por Hair, Sarstedt, Matthews e Ringle (2016) e Hair et al. (2016), confirmando a validade convergente (ver apêndice A). Todos os construtos apresentaram variância média extraída (AVE) acima de 0,5 (Fornell \& Larcker, 1981).

A confiabilidade de um modelo pode ser avaliada pelo alfa de Cronbach ou pela confiabilidade composta, e no contexto de modelagem de equaçóes estruturais e PLS-PM, o indicador alfa de Cronbach mostra-se sensível ao número de itens na variável, sendo a confiabilidade composta considerada mais apropriada, conforme Hair et al. (2016). A confiabilidade dos construtos demonstrou-se adequada com valores de confiabilidade composta (CR) acima do limite de 0,60 (Chin, 1998; Hair et al., 2017; Henseler, Ringle, \& Sinkovics, 2009). Pela Tabela 3, observou-se que os valores na diagonal (raiz quadrada da variância média extraída) foram maiores que os valores fora da diagonal (correlaçóes), demonstrando a existência de validade discriminante (Hair et al., 2017; Ringle, Bido, \& Silva, 2014). Os resultados do teste HTMT (heterotrait-monotrait ratio) indicaram valores abaixo de 0,85 , confirmando a validade discriminante, como recomendado por Henseler, Ringle, e Sarstedt (2015).

Tabela 3. Matriz de correlaçóes entre os construtos e demais mensuraçóes.

\begin{tabular}{|c|c|c|c|c|}
\hline Construto & 1 & 2 & 3 & 4 \\
\hline 1 - Dinamismo ambiental & 0,75 & & & \\
\hline $2-$ SIS & 0,40 & 0,87 & & \\
\hline 3 - Inovação de exploration & 0,55 & 0,60 & 0,80 & \\
\hline 4 - Inovação de exploitation & 0,48 & 0,67 & 0,71 & 0,76 \\
\hline Variância média extraída (AVE) & 0,56 & 0,76 & 0,64 & 0,58 \\
\hline Confiabilidade composta (CR) & 0,79 & 0,94 & 0,90 & 0,87 \\
\hline Média & 3,96 & 4,81 & 4,23 & 5,01 \\
\hline Desvio padrão & 1,38 & 1,14 & 1,31 & 1,18 \\
\hline Alfa de Cronbach (CA) & 0,67 & 0,92 & 0,86 & 0,82 \\
\hline
\end{tabular}

Nota. Os valores na diagonal são a raiz quadrada da AVE (primeiro bloco). Intervalos desejáveis de métricas indicativas de qualidade do modelo $=$ AVE $>0,5$ (Fornell $\&$ Larcker, 1981); CA e CC variam entre 0,6 e 0,9 (Chin, 1998; Hair et al., 2017; Henseler et al., 2009). Fonte: Elaborado pelos autores.

\section{Modelo de estrutural}

A operacionalização do modelo inclui a avaliação do efeito de moderação do dinamismo ambiental, bem como a inclusão das variáveis de controle (tamanho da empresa e setor); ver Tabela 4. Para mensurar o tamanho do efeito $\left(\mathrm{f}^{2}\right)$ foi seguida a recomendação da literatura metodológica (Cohen, 1988; Hair, Sarstedt, Ringle, \& Gudergan, 2018; Henseler et al., 2009), a qual sugere que os valores do tamanho do efeito de $0,02,0,15$ e 0,35 são respectivamente pequeno, médio e grande. 
Tabela 4. Coeficientes de regressão padronizados dos modelos estruturais com todas as variáveis.

\begin{tabular}{|c|c|c|c|c|c|c|c|}
\hline \multirow[b]{2}{*}{ Relaçôes } & \multicolumn{3}{|c|}{ Sem MLMV } & \multicolumn{3}{|c|}{ Com MLMV } & \multirow[b]{2}{*}{$\mathrm{R}^{2}$} \\
\hline & $\mathrm{f}^{2}$ & $\begin{array}{l}\text { Coeficiente de } \\
\text { caminho }\end{array}$ & Valor-p & $\mathrm{R}^{2}$ & $\begin{array}{l}\text { Coeficiente de } \\
\text { caminho }\end{array}$ & Valor-p & \\
\hline SIS $\rightarrow$ Exploration & 0,331 & 0,455 & 0,000 & & 0,460 & 0,000 & \\
\hline Dinamismo $\rightarrow$ Exploration & 0,235 & 0,369 & 0,000 & & 0,370 & 0,000 & \\
\hline SIS * Dinamismo $\rightarrow$ Exploration & 0,043 & 0,135 & 0,000 & $51,70 \%$ & 0,137 & 0,004 & $51,80 \%$ \\
\hline Setor $\rightarrow$ Exploration & 0,041 & $-0,142$ & 0,010 & & $-0,147$ & 0,004 & \\
\hline Funcionários $\rightarrow$ Exploration & 0,002 & 0,034 & 0,459 & & 0,034 & 0,459 & \\
\hline SIS $->$ Exploitation & 0,484 & 0,550 & 0,000 & & 0,531 & 0,000 & \\
\hline Dinamismo $\rightarrow$ Exploitation & 0,115 & 0,259 & 0,000 & & 0,255 & 0,000 & \\
\hline SIS * Dinamismo $\rightarrow$ Exploitation & 0,000 & 0,009 & 0,850 & $51,50 \%$ & 0,004 & 0,928 & $52,30 \%$ \\
\hline Setor $\rightarrow$ Exploitation & 0,037 & $-0,135$ & 0,083 & & $-0,118$ & 0,124 & \\
\hline Funcionários $\rightarrow$ Exploitation & 0,000 & $-0,007$ & 0,885 & & $-0,008$ & 0,860 & \\
\hline
\end{tabular}

Nota. O setor foi medido por dois indicadores formativos (dummy) para representar as seguintes categorias: Agronegócio, Governo, Indústria e Serviços. A significância foi estimada por bootstrap com $\mathrm{n}=256$ casos e 5000 repetiçôes no SmartPLS v3. A mensuração do método da coleta de viés foi realizada com a inclusão dos indicadores MLMV. Intervalos desejáveis de métricas indicativas de qualidade do modelo = $\mathrm{f}^{2}$ com tamanho do efeito de 0,02 (pequeno), 0,15 (médio) ou 0,35 (grande) (Cohen, 1988 ; Henseler et al., 2009); valor-p < 0,05 (Hair et al., 2017); $\mathrm{R}^{2}$ com valores de 0,67 (substancial), 0,33 (moderado) ou 0,19 (fraco) (Chin, 1998; Henseler et al., 2009). Fonte: Elaborado pelos autores.

A possibilidade de heterogeneidade da amostra (constituída por empresas de variados setores da economia e de portes diferentes) foi uma das justificativas para a inclusão das variáveis de controle de setor e tamanho (número de funcionários) da organização no modelo estudado. A literatura de sistemas de informação reporta estudos (Benitez, Castillo, Llorens, \& Braojos, 2018; Benitez, Llorens, \& Braojos, 2018) que examinaram o impacto da tecnologia da informação (TI) na inovação, utilizando técnica estatística de PLS-PM com uso de variáveis de controle de setor e tamanho. Nos resultados da pesquisa, apenas a variável de controle de setor na variável dependente de inovação de exploration apresentou significância estatística (valor-p < 0,01) com coeficiente de caminho negativo.

A hipótese H1a foi suportada $\left(\beta=0,455\right.$; $\mathrm{f}^{2}=0,331$; valor-p < 0,001; $\mathrm{R}^{2}=51,7 \%$ ) e confirmou que o SIS influencia a inovação de exploration, com efeito grande e significante. A hipótese H1b foi corroborada $\left(\beta=0,550\right.$; $\mathrm{f}^{2}=0,484$; valor-p $\left.<0,001 ; R^{2}=51,5 \%\right)$ e confirmou que o SIS influencia a inovação de exploitation.

Comparou-se a diferença dos efeitos de caminho nas relaçóes entre o SIS e a inovação de exploration e entre o SIS e a inovação de exploitation, verificando-se uma diferença de 0,095 nos coeficientes estruturais.

Para verificar a existência de significância estatística nesta diferença entre coeficientes de caminho de uma mesma amostra, utilizou-se o framework metodológico para testar diferenças entre coeficientes de caminho sugerido por Rodríguez-Entrena, Schuberth e Gelhard (2018). A significância estatística foi estimada usando a técnica de bootstrap com 5.000 subamostras, com o intervalo de confiança de $95 \%$. Verificou-se por meio dos testes de intervalo de confiança de standard/Student's t, percentile bootstrap e standard bootstrap que os valores entre os intervalos de confiança passaram por zero, demonstrando a não existência de significância estatística (valor-p > 0,05) para a diferença de 0,095 (17\%) entre os coeficientes de caminho das relaçóes de SIS -> exploration e SIS -> exploitation; ver Tabela 5.

Tabela 5. Teste de significância estatística entre duas estimativas de coeficientes de caminho pelo PLS-PM.

\begin{tabular}{lcc}
\hline $\begin{array}{l}\text { Tipo de intervalo de } \\
\text { confiança }(\alpha=5 \%)\end{array}$ & Limite inferior & Limite superior \\
\hline Standard & $-0,089$ & $-0,077$ \\
Percentile & $-0,196$ & 0,019 \\
Basic & $-0,194$ & 0,021 \\
\hline
\end{tabular}

Nota. Fonte: Elaborado pelos autores.

A hipótese $\mathrm{H} 2 \mathrm{~b}$ não foi suportada $\left(\beta=0,009 ; \mathrm{f}^{2}=0,00\right.$; valor-p > 0,05; $\left.\mathrm{R}^{2}=51,5 \%\right)$, pois a variável contínua de dinamismo não apresentou moderação estatisticamente significante na relação entre SIS e inovação de exploitation. Contudo, na relação entre SIS e inovaçáo de exploration, a variável de dinamismo apresentou relação positiva e grande efeito com significância estatística $\left(\beta=0,135\right.$; $\mathrm{f}^{2}=0,043$; valor-p < 0,001; $\mathrm{R}^{2}=51,7 \%$ ), suportando a hipótese $\mathrm{H} 2 \mathrm{a}$. Como interpretação deste achado, pode-se considerar que à medida que o dinamismo ambiental se torna mais alto (por exemplo, com aumento de um desvio padrão), a relação entre SIS e exploration aumentará com o tamanho do termo de interaçáa, sendo obtido o valor de coeficiente de caminho de $0,590(0,455+0,135)$, representando um aumento de $23 \%$ nesta relação, conforme ilustrado na Figura 3. 


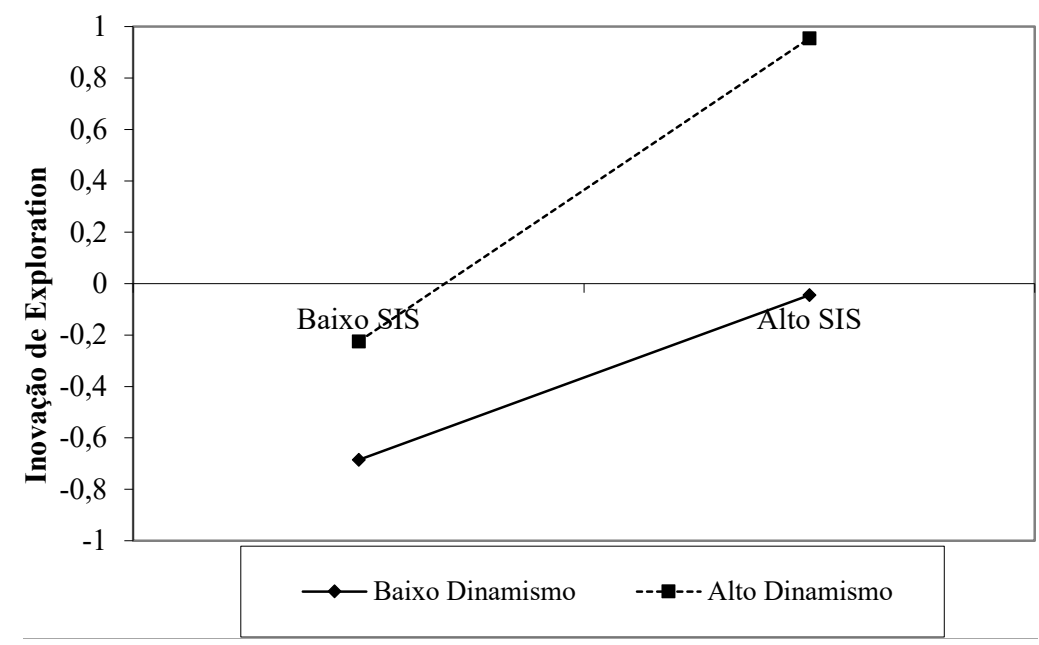

Figura 3. Gráfico do efeito de moderação do dinamismo na relação entre SIS e exploration, de acordo recomendaçóes de Dawson (2014).

Fonte: Elaborado pelos autores com uso do software SmartPLS v.3.

\section{Pós-análise (post hoc analysis)}

O estudo pós-análise permitiu identificar e interpretar a possível 'heterogeneidade não observada' na relação entre o SIS e a inovação de exploration e exploitation, verificando a existência de fatores que não estão incluídos na análise original, e que podem explicar as diferenças entre vários grupos de empresas. Utilizou-se a técnica de mistura finalizada (FIMIX-PLS), conforme recomendado por Hair et al. (2016) e Matthews, Sarstedt, Hair e Ringle (2016) sobre as análises do PLS-PM.

Para identificar o número de segmentos não observáveis foi utilizado o algoritmo FIMIX-PLS (software SmartPLS v3) (Ringle, Wende, \& Becker, 2015), executado 10 vezes para $\mathrm{g}=2-5$ segmentos, usando os critério de informaçáo Akaike (AIC), AIC modificado com fator 3 (AIC3), critério de informação bayesiano (BIC), AIC de consistência (CAIC), critério Hannan-Quinn (HQ) e estatística normalizada de entropia (EN), que apresentaram critérios satisfatórios para a seleção dos segmentos.
Os indicadores que apresentam menores valores em certos critérios de informaçáo indicam a melhor solução de segmentos e evidenciam o valor de EN acima de 0,50 (Hair et al., 2018). Conforme os autores, o primeiro critério verifica se a combinaçáo dos valores de AIC3 e CAIC são menores por segmento. Como segundo critério, devem ser considerados os menores valores na combinaçáo do conjunto AIC3 e BIC. Como terceiro critério, verificam-se os menores valores para a combinaçáo de AIC4 e BIC. Caso nenhum dos critérios anteriores seja atendido, a recomendação geral é que se escolha o menor valor do segmento indicado pelo AIC e mais segmentos daquele indicado pelo critério do MDL5.

Além dos critérios informados, o tamanho mínimo da amostra deve ser atendido, que neste estudo foi identificado com o mínimo de 50 casos, ou seja, o número máximo de caminhos estruturais que apontam para a variável dependente (5) multiplicado por 10, segundo critérios utilizados na literatura metodológica (Hair et al., 2017). Os critérios apresentados na Tabela 6 especificam que a solução com dois segmentos é a mais adequada, e assim prosseguiu a abordagem de segmentação orientada para predição (PLS-POS).

Tabela 6. Índices de ajuste para uma solução de um a cinco segmentos.

\begin{tabular}{|c|c|c|c|c|c|}
\hline Critérios & & & de segmentos & & \\
\hline & 1 & 2 & 3 & 4 & 5 \\
\hline AIC & $1.189,43$ & $1.165,65$ & $1.143,62$ & $1.148,00$ & $1.151,54$ \\
\hline AIC3 & $1.193,43$ & $1.174,65$ & $1.157,62$ & $1.167,00$ & $1.175,54$ \\
\hline AIC4 & $1.197,43$ & $1.183,65$ & $1.171,62$ & $1.186,00$ & $1.199,54$ \\
\hline BIC & $1.203,61$ & $1.197,56$ & $1.193,25$ & $1.215,35$ & $1.236,62$ \\
\hline CAIC & $1.207,61$ & $1.206,56$ & $1.207,25$ & $1.234,35$ & $1.260,62$ \\
\hline HQ & $1.195,13$ & $1.178,49$ & $1.163,58$ & $1.175,09$ & $1.185,76$ \\
\hline MDL5 & $1.292,28$ & $1.397,19$ & $1.503,78$ & $1.636,79$ & $1.768,96$ \\
\hline $\mathrm{EN}$ & & 0,28 & 0,53 & 0,50 & 0,46 \\
\hline
\end{tabular}

Nota. AIC - critério de informação de Akaike; AIC3 - AIC com fator 3 modificado; AIC4 - AIC com fator 4 modificado; BIC - critério de informaçáo bayesiano; CAIC - AIC de consistência; HQ - critério de Hannan-Quinn; MDL5 - comprimento mínimo da descriçáo com fator 5; EN - estatística de entropia (normalizada). Fonte: Elaborado pelos autores. 
Tabela 7. Tamanho relativo dos segmentos.

\begin{tabular}{cccccc}
\hline $\begin{array}{c}\text { Número de } \\
\text { segmentos }\end{array}$ & \multicolumn{5}{c}{ Tamanho relativo dos segmentos } \\
\hline & 1 & 2 & 3 & 4 & 3 \\
2 & 0,519 & 0,481 & & & \\
3 & 0,559 & 0,283 & 0,158 & & \\
4 & 0,504 & 0,267 & 0,149 & 0,08 & \\
5 & 0,294 & 0,233 & 0,226 & 0,175 & 0,072 \\
\hline
\end{tabular}

Nota. Fonte: Elaborado pelos autores.

Para avaliar as diferenças dos segmentos e soluçóes alternativas, executou-se o algoritmo PLS-POS (software SmarPLS v3) (Ringle et al., 2015) para os dois segmentos sugeridos pelo FIMIX-PLS, e foram comparados os valores de $\mathrm{R}^{2}$ do PLS-POS das variáveis dependentes $\left(\mathrm{R}^{2}\right.$ original, $\mathrm{R}^{2}$ 'segmento 1 ', $\mathrm{R}^{2}$ 'segmento 2' e média dos $\mathrm{R}^{2}$ do PLSPOS). Na Tabela 8 foram informadas as diferenças entre o $\mathrm{R}^{2}$ da amostra original e os demais $\mathrm{R}^{2}$ ('segmento 1 ', 'segmento 2', e a média do PLS-POS), requisitando interpretaçóes complementares entre os grupos, como recomendado pela literatura (Hair et al., 2018).

Para a avaliação do dinamismo ambiental nesta seção de pós-análise, foi utilizado o valor médio desta variável na atribuição de grupos com baixa e alta presença de incerteza ambiental, com base no procedimento adotado pelo estudo do SIS e dinamismo realizado por Yayla e Hu (2012). Dessa forma, valores acima da média da respectiva dimensão foram considerados de alta presença de dinamismo ('alto dinamismo', 147 casos), e valores abaixo da média foram considerados de baixa presença de dinamismo ('baixo dinamismo', 109 casos).

$\mathrm{Na}$ Tabela 9, comparando a contagem de células, descobriu-se que a segunda segmentação ('segmento 2' do PLS-POS) foi a melhor combinação para as variáveis de dinamismo ambiental, setor e tamanho de empresa, apresentando sobreposição adequada de $65 \%$ do tamanho da cobertura da amostra, conforme recomendação da literatura (Hair et al., 2018).

Tabela 8. PLS-POS solução por segmento.

\begin{tabular}{lllll}
\hline $\begin{array}{c}\text { Variável } \\
\text { dependente }\end{array}$ & $\begin{array}{c}\mathrm{R}^{2} \text { da } \\
\text { amostra } \\
\text { original }\end{array}$ & $\begin{array}{c}\mathrm{R}^{2} \text { do } \\
\text { segmento 1 }\end{array}$ & $\begin{array}{c}\mathrm{R}^{2} \text { do } \\
\text { segmento 2 }\end{array}$ & $\begin{array}{c}\text { Média dos } \\
\mathrm{R}^{2} \text { do PLS- } \\
\text { POS }\end{array}$ \\
\hline Exploitation & 0,447 & 0,281 & 0,732 & 0,572 \\
Exploration & 0,374 & 0,315 & 0,714 & 0,573 \\
\hline
\end{tabular}

Nota. Intervalos desejáveis de métricas indicativas de qualidade do modelo $=\mathrm{R}^{2}$ com os valores de 0,67 (substancial), 0,33 (moderado) ou 0,19 (fraco) (Chin, 1998; Henseler et al., 2009). Fonte: Elaborado pelos autores.

Tabela 9. Tabulação cruzada por segmento PLS-POS e variáveis de moderação.

\begin{tabular}{|c|c|c|c|c|c|c|c|}
\hline \multicolumn{2}{|c|}{ Variáveis } & \multicolumn{3}{|c|}{ Tamanho da amostra por segmento PLS-POS } & \multicolumn{2}{|c|}{$\begin{array}{l}\text { Percentual da amostra por } \\
\text { segmento PLS-POS }\end{array}$} & \multirow{2}{*}{$\begin{array}{c}\text { Total } \\
\%\end{array}$} \\
\hline & & 1 & 2 & Total & 1 & 2 & \\
\hline \multirow{2}{*}{ Dinamismo } & Alto & 40 & 107 & 147 & $15 \%$ & $42 \%$ & $57 \%$ \\
\hline & Baixo & 51 & 58 & 109 & $20 \%$ & $23 \%$ & $43 \%$ \\
\hline \multirow{4}{*}{ Setores } & Agronegócio & 5 & 6 & 11 & $2 \%$ & $2 \%$ & $4 \%$ \\
\hline & Indústria & 34 & 59 & 93 & $13 \%$ & $23 \%$ & $36 \%$ \\
\hline & Serviço & 48 & 96 & 144 & $18 \%$ & $38 \%$ & $56 \%$ \\
\hline & Governo & 4 & 4 & 8 & $2 \%$ & $2 \%$ & $3 \%$ \\
\hline \multirow{6}{*}{$\begin{array}{l}\text { Tamanho da empresa } \\
\text { (n. }{ }^{\circ} \text { de funcionários) }\end{array}$} & Até 9 & 10 & 13 & 23 & $4 \%$ & $5 \%$ & $9 \%$ \\
\hline & 10 até 49 & 8 & 21 & 29 & $3 \%$ & $8 \%$ & $11 \%$ \\
\hline & 50 até 99 & 13 & 28 & 41 & $5 \%$ & $11 \%$ & $16 \%$ \\
\hline & 100 até 249 & 17 & 19 & 36 & $7 \%$ & $7 \%$ & $14 \%$ \\
\hline & 250 até 499 & 13 & 11 & 24 & $4 \%$ & $4 \%$ & $9 \%$ \\
\hline & Acima de 500 & 30 & 73 & 103 & $12 \%$ & $29 \%$ & $40 \%$ \\
\hline
\end{tabular}

Nota. O critério determinado para a definição de grupos de 'alto dinamismo' e 'baixo dinamismo' seguiu o procedimento adotado por Yayla e Hu (2012). Fonte: Elaborado pelos autores.

A partir das diferenças apresentadas por segmento PLSPOS (Tabelas 8 e 9), calcularam-se os modelos estruturais para o 'segmento 1' e o 'segmento 2'. A Tabela 10 descreve os resultados dos modelos estruturais com significância estatística (valor-p < 0,05), validade convergente e confiabilidade de acordo com Hair et al. (2017). Além disso, foi executado o método PLS-MGA para comparar os coeficientes de caminho dos modelos com a amostra original, identificando diferenças na relação entre SIS e inovação de exploitation $(|\mathrm{p} 1-\mathrm{p} 2|=$ $0,326$; valor- $t=6,185$; valor- $\mathrm{p}<0,001)$, e na relaçâao entre SIS e inovação de exploration $(|\mathrm{p} 1-\mathrm{p} 2|=0,284$; valor- $\mathrm{t}=10,463$; valor-p $<0,001)$. 
Tabela 10. Resultados agregados por grupo.

\begin{tabular}{|c|c|c|c|c|c|}
\hline Grupo & Modelos estruturais & Coeficiente estrutural & Erro padrão & Valor-p & $\mathrm{R}^{2}$ \\
\hline \multirow{2}{*}{ Original } & SIS $->$ Exploitation & 0,668 & 0,032 & 0,000 & $44,7 \%$ \\
\hline & SIS $\rightarrow$ Exploration & 0,612 & 0,036 & 0,000 & $37,4 \%$ \\
\hline \multirow{2}{*}{1} & SIS $->$ Exploitation & 0,530 & 0,063 & 0,000 & $28,1 \%$ \\
\hline & SIS -> Exploration & 0,561 & 0,246 & 0,022 & $31,5 \%$ \\
\hline \multirow{2}{*}{2} & SIS $->$ Exploitation & 0,856 & 0,018 & 0,000 & $73,2 \%$ \\
\hline & SIS $->$ Exploration & 0,845 & 0,018 & 0,000 & $71,4 \%$ \\
\hline
\end{tabular}

Nota. Intervalos desejáveis de métricas indicativas de qualidade do modelo = valor-p < 0,05 (Hair et al., 2017); $\mathrm{R}^{2}$ com valores de 0,67 (substancial), 0,33 (moderado) ou 0,19 (fraco) (Chin, 1998; Henseler et al., 2009). Fonte: Elaborado pelos autores.

\section{DISCUSSÃO E CONCLUSÃO}

O estudo examinou as relações entre os construtos dos sistemas de informação estratégicos (SIS) e inovação sobre a incerteza ambiental. Os resultados demonstraram que o modelo proposto tem alto poder explicativo, superior a $50 \%$ de explicação em todas as variáveis endógenas do modelo.

O resultado da pesquisa identificou efeitos positivos do SIS sobre a inovação de exploitation e sobre inovação de exploration, confirmando as hipóteses H1a e H1b. Em outras palavras, os processos de inovaçáo são habilitados pelos sistemas de informação estratégicos que apoiam o processo evolutivo da inovação na empresa, aumentando a eficiência operacional nos processos de negócios e geração de inovação incremental nos produtos/serviços, e apoiando as iniciativas de inovação radical, como, por exemplo, o lançamento de novos produtos/serviços.

A hipótese H2b não foi corroborada. Não foi confirmada a moderação do dinamismo na relação entre o SIS e a inovação de exploitation. O resultado confirmou estudos anteriores de SI (Leidner et al., 2011; Mikalef \& Pateli, 2017) que demonstraram que nos recursos de TI em determinadas configuraçóes de incerteza ambiental, a relaçáo entre a TI e a capacidade da organização em atender as necessidades de inovação do mercado náo se altera. Portanto, esse resultado sugere que, mesmo que o mercado seja altamente dinâmico, as empresas com a estratégia de inovação incremental não sofrerão alteração pela influência do SIS. Conforme Porter (1990), Mintzberg, Ahlstrand, and Lampel (2009) e Miles, Snow, Meyer, e Coleman (1978), empresas que adotam a estratégia de inovação incremental sofrem pressão do ambiente externo para oferecer produtos e/ou serviços com preços competitivos e, portanto, essas empresas possuem foco na produtividade (eficiência e eficácia) para oferecer produtos e/ou serviços com preços mais baixos. Assim, a incerteza do ambiente pelo dinamismo pode não apresentar influência na relação entre a TI e a inovação de exploitation.
O estudo mostrou que o SIS habilita a organização em ambientes com alta taxa de mudança e imprevisibilidade, disponibilizando de forma rápida informaçôes para compreender e tomar decisóes neste ambiente em constante transformação, essenciais para as iniciativas de inovação radical, dando suporte à hipótese $\mathrm{H} 2 \mathrm{a}$. Ou seja, o resultado sugere que as empresas que adotam a estratégia de inovação de exploration sofrem pressão do ambiente externo para criar novos produtos e serviços, ao contrário da hipótese $\mathrm{H} 2 \mathrm{~b}$. Contudo, o SIS contribuiu para ambos os tipos de inovação de exploitation e exploration, principalmente para as empresas que adotam a estratégia de inovação de exploration.

Dessa forma, o SIS pode ser uma alternativa para empresas promoverem iniciativas emergentes de inovação radical, alinhadas ao direcionamento deliberativo dos objetivos estratégicos da empresa, conforme teorizado como essencial na abordagem da estratégia como prática (Bodwell \& Chermack, 2010; Bolisani \& Bratianu, 2017), e corroborando recentes pesquisas no campo de SI que demonstraram a relevância do papel da TI para as organizaçóes enfrentarem os efeitos da incerteza ambiental (Dameron et al., 2015; George et al., 2014; Rouhani et al., 2016; Yoshikuni \& Albertin, 2017).

Além disso, o estudo de pós-análise examinou a heterogeneidade náo observada da amostra (em dois grupos segmentados do modelo original da pesquisa); foi demonstrado que em 165 empresas do 'segmento 2' (maior representatividade das variáveis observáveis, com $38 \%$ do setor de serviços, $29 \%$ de empresas acima de 500 funcionários e $42 \%$ de presença do alto dinamismo) houve incremento no coeficiente de caminho das relaçóes entre o SIS e a inovação de exploitation $\left(\beta=0,856\right.$; valor-p $\left.<0,001 ; \mathrm{R}^{2}=73,2 \%\right)$ e entre o SIS e a inovação de exploration $(\beta=0,845$; valor-p < 0,$\left.001 ; R^{2}=71,4 \%\right)$. Portanto, tais resultados demonstraram que em determinados clusters (setor, tamanho e alto grau de dinamismo) o SIS possui efeitos fortes e positivos em habilitar a inovação de exploitation e exploration sob a influência da incerteza ambiental, em linha com estudos de SI que apresentaram a importância de uma análise 
segmentada da amostra para a identificaçáo da agregaçáo de valor da TI no negócio (Mikalef \& Pateli, 2017; Wilden \& Gudergan, 2014; Yoshikuni, Lucas, \& Albertin, 2019)

\section{Implicações para a teoria}

O estudo contribuiu para a extensão do conhecimento da literatura de sistemas de informaçáo e estratégia como prática ao expor como a adoção do SIS pode ser uma alternativa para as organizaçóes brasileiras integrarem as abordagens deliberativa e emergente da estratégia como prática, a fim de promover inovação em um ambiente de incerteza ambiental.

Portanto, o estudo demonstrou que fundamentos teóricos apresentados pela abordagem da estratégia como prática, que muitas vezes podem ser vagos e abstratos em estudos conceituais, podem ser decompostos em atividades específicas que podem ser mensuradas com a incorporação das aplicaçôes tecnológicas. Em outras palavras, o artigo examinou como as aplicaçóes de SI são incorporadas ao processo da estratégia, cuja agregação e sinergias abrangem uma capacidade organizacional de promover a inovação de exploitation e exploration. Dessa forma, seguindo os questionamentos de como a TI pode agregar valor à estratégia, especialmente em ambientes dinâmicos e turbulentos (Kohli \& Grover, 2008), o estudo identificou e examinou como as aplicaçóes de SI são fundamentais para promover a estratégia como prática (atendendo a recentes estudos de SI e estratégia como prática) (Whittington, 2014) e para habilitar a inovação na organização, conforme mencionado por Marabelli e Galliers (2017).

Além disso, o estudo contribuiu para a literatura na abordagem da estratégia como prática, ao mostrar que a ubiquidade da TI por meio do SIS incorporado ao processo de estratégia habilita a inovaçáo, rompendo com o paradoxo de rigidez do planejamento estratégico de não influenciar a inovaçáo, conforme mencionado em estudos de planejamento estratégico e inovação (Arend et al., 2017; Song, Im, Van Der Bij, \& Song, 2011). Assim, o estudo contribui para o conhecimento da literatura de sistema de informaçáo e estratégia como prática ao apresentar uma alternativa viável para as empresas responderem com flexibilidade e agilidade aos desafios do dinamismo externo, e para desenvolverem iniciativas estratégicas de inovação radical e incremental.

\section{Implicações práticas}

$\mathrm{Na}$ prática, os resultados do estudo forneceram aos executivos a compreensão clara sobre como as aplicaçóes de SI incorporadas ao processo de planejamento estratégico habilitam na organização a estratégia como prática (estratégia deliberativa e emergente) com o objetivo de promover a inovaçáo de exploitation e exploration. Assim, o estudo demonstrou a importância de as empresas investirem em aplicações de SI para promover a prática da estratégia na organizaçáo, com o objetivo de alavancar a inovaçáo e enfrentar a incerteza ambiental.

Portanto, a incorporação das aplicaçóes de SI nas atividades da estratégia não resultará apenas na produtividade e no aumento da velocidade das atividades internas para habilitar a inovação incremental, mas também permitirá que as empresas desenvolvam inovaçáo radical frente às oportunidades de mercado nestes ambientes, previamente à concorrência.

Além disso, com base nos resultados pós-análise, ficou evidenciado que as aplicaçóes de SI incorporadas no processo de estratégia permitem às organizações (em determinadas características de setor, tamanho e incerteza ambiental) habilitar as diferentes posturas de inovação de exploitation e exploration sob as condiçóes de alta taxa de dinamismo.

\section{Limitação e estudos futuros}

Apesar do rigor metodológico seguido pelos pesquisadores na estruturação do instrumento de coleta, qualificação dos respondentes, e no tratamento dos dados, a amostragem não probabilística por conveniência é considerada uma limitação neste estudo, não permitindo generalizaçóes.

Como estudos futuros, sugerem-se pesquisas que possam investigar categorias que compuseram os subgrupos heterogêneos identificados na seção de pós-análise; ampliar a investigação da relação entre o SIS e a inovação ao incluir novos construtos que habilitam a organização frente aos desafios do dinamismo; investigar se a inovação ambidestra (inovação simultânea de exploration e exploitation) pode ser habilitada pelo SIS em condições de incerteza ambiental do dinamismo; desenvolver estudos longitudinais como forma de identificar a possível causalidade do SIS nas iniciativas de inovação. São questionamentos em aberto na literatura que poderáo ser investigados em estudos por vir. 


\section{REFERÊNCIAS}

Aguirre-Urreta, M., \& Rönkkö, M. (2015). Sample size determination and statistical power analysis in PLS Using R: An annotated tutorial. Communications of the Association for Information Systems, 36(3), 33-51. https://doi.org/10.17705/1CAIS.03603

Arend, R. J., Zhao, Y. L., Song, M., \& Im, S. (2017). Strategic planning as a complex and enabling managerial tool. Strategic Management Journal, 38, 1741-1752. https://doi.org/10.1002/smj.2420.

Arvidsson, V., Holmström, J., \& Lyytinen, K. (2014). Information systems use as strategy practice : A multi-dimensional view of strategic information system implementation and use. Journal of Strategic Information Systems, 23(1), 45-61. https://doi.org/10.1016/j.jsis.2014.01.004

Barbero, J. L., Ramos, A., \& Chiang, C. (2017). Restructuring in dynamic environments: A dynamic capabilities perspective. Industrial and Corporate Change, 26(4), dtw042. https://doi.org/10.1093/icc/dtw042

Benitez, J., Castillo, A., Llorens, J., \& Braojos, J. (2018). IT-enabled knowledge ambidexterity and innovation performance in small U.S. firms: The moderator role of social media capability. Information and Management, 55(1), 131-143. https://doi.org/10.1016/j.im.2017.09.004

Benitez, J., Llorens, J., \& Braojos, J. (2018). How information technology influences opportunity exploration and exploitation firm's capabilities. Information \& Management, 55(4), 508-523. https://doi.org/10.1016/j.im.2018.03.001

Benner, M. J., \& Tushman, M. L. (2003). Exploitation, exploration, and process management: The productivity dilemma revisited. Academy of Management Review, 28(2), 238256. https://doi.org/10.5465/AMR.2003.9416096

Bisquerra, R., Sarriera, J. C., \& Martínez, F. (2004). Introdução à estatística: Enfoque informático com o pacote estatistico SPSS. São Paulo: Artmed.

Bodwell, W., \& Chermack, T. J. (2010). Organizational ambidexterity: Integrating deliberate and emergent strategy with scenario planning. Technological Forecasting and Social Change, 77(2), 193-202. https://doi.org/10.1016/j.techfore.2009.07.004

Bolisani, E., \& Bratianu, C. (2017). Knowledge strategy planning: An integrated approach to manage uncertainty, turbulence, and dynamics. Journal of Knowledge Management, 21(2), 233-253. https://doi.org/10.1108/JKM-02-2016-0071

Chan, Y. E., \& Huff, S. L. (1992). Strategy: An information systems research perspective. The Journal of Strategic Information Systems, 1(4), 191-204. https://doi.org/10.1016/0963-8687(92)90035-U

Chan, Y. E., Sabherwal, R., \& Thatcher, J. B. (2006). Antecedents and outcomes of strategic IS alignment: an empirical investigation. IEEE Transactions on Engineering Management, 53(1), 27-47. https://doi.org/10.1109/TEM.2005.861804
Chen, D. Q., Mocker, M., Preston, D. S., \& Teubner, A. (2010). Information Systems Strategy: Reconceptualization, Measurement, and Implications. MIS Quarterly, 34(2), 233-259. https://doi.org/10.2307/20721426

Chen, H., Zeng, S., Lin, H., \& Ma, H. (2017). Munificence, dynamism, and complexity: How industry context drives corporate sustainability. Business Strategy and the Environment, 26(2), 125-141. https://doi.org/10.1002/bse.1902

Chen, Y., Wang, Y., Nevo, S., Jin, J., Wang, L., \& Chow, W. S. (2014). IT capability and organizational performance: The roles of business process agility and environmental factors. European Journal of Information Systems, 23(3), 326-342. https://doi.org/10.1057/ejis.2013.4

Chin, W. W. (1998). The partial least squares approach to structural equation modeling. In G. A. Marcoulides (Ed.), Modern Methods for Business Research (pp. 295-336). Lawrence Erlbaum Associates. https://doi.org/10.1057/ejis.2013.4

Chin, W. W., Thatcher, J. B., Wright, R. T., \& Steel, D. (2013). Controlling for common method variance in PLS analysis: the measured latent marker variable approach (pp. 231239). In H Abdi, W. W. Chin, V. E. Vinzi, Russolillo, G. \& L. Trinchera (Eds.), New Perspectives in Partial Least Squares and Related Methods. New York: Springer.

Chuang, S. H., \& Lin, H. N. (2017). Performance implications of information-value offering in e-service systems: Examining the resource-based perspective and innovation strategy. Journal of Strategic Information Systems, 26(1), 22-38. https://doi.org/10.1016/j.jsis.2016.09.001

Cohen, J. (1988). Statistical power analysis for the behavioral sciences (2nd ed.). Mahwah, NJ: Lawrence Erlbaum Associates.

Cousineau,D., \&Chartier,S.(2017).Outliersdetectionand treatment: A review. International Journal of Psychological Research, 3(1), 58. https://doi.org/10.21500/20112084.844

Dameron, S., Lê, J. K., \& Lebaron, C. (2015). Materializing Strategy and Strategizing Materials: Why Matter Matters. British Journal of Management, 26, S1-S12. https://doi.org/10.1111/1467-8551.12084

Davenport, T. H., Harris, J. G., \& Morison, R. (2010). Analytics at work: Smarter decisions, better results. Brighton: Harvard Business Press.

Dawson, J. F. (2014). Moderation in management research: What, why, when, and how. Journal of Business and Psychology, 29(1), 1-19. https://doi.org/10.1007/s10869-013-9308-7

Etikan, I., Musa, S. A., \& Alkassim, R. S. (2016). Comparison of Convenience Sampling and Purposive Sampling. American Journal of Theoretical and Applied Statistics, 5(1), 1-4. https://doi.org/10.11648/j.ajtas.20160501.11

Fornell, C., \& Larcker, D. F. (1981). Evaluating structural equation models with unobservable variables and measurement error. Journal of Marketing Research, 18(1), 39-50. https://doi.org/10.2307/3151312 
Fuller, C. M., Simmering, M. J., Atinc, G., Atinc, Y., \& Babin, B. J. (2016). Common methods variance detection in business research. Journal of Business Research, 69(8), 3192-3198. https://doi.org/10.1016/j.jbusres.2015.12.008

Galliers, R. D. (2011). Further developments in information systems strategizing: Unpacking the concept. In R. D. Galliers \& W. L. Currie (Eds.), The oxford handbook of management information systems: Critical perspectives and new directions (pp. 329-345). Oxford: Oxford University Press.

George, G., Haas, M. R., \& Pentland, A. (2014). From the editors: Big data and management. Academy of Management Journal, 57(2), 321-326. https://doi.org/10.5465/amj.2014.4002

Goodhue, D., Lewis, W., \& Thompson, R. (2007). Statistical power in analyzing interaction effects: Questioning the advantage of PLS with product indicators. Information Systems Research, 18(2), 211-227. https://doi.org/10.1287/isre.1070.0123

Gupta, A. K., Smith, K. G., \& Shalley, C. E. (2006). The interplay between exploration and exploitation. Academy of Management Journal, 49(4), 693-706. https://doi.org/10.5465/AMJ.2006.22083026

Hair, J. F., Hult, G., Ringle, C., \& Sarstedt, M. (2017). A primer on partial least squares structural equation modeling (PLSSEM) (2a ed.). Thousand Oaks, CA: Sage Publications.

Hair, J. F., Sarstedt, M., Matthews, L. M., \& Ringle, C. M. (2016). Identifying and treating unobserved heterogeneity with FIMIX-PLS : Part I method. European Business Review, 28(1), 63-76. https://doi.org/10.1108/EBR-09-2015-0094

Hair, J. F., Sarstedt, M., Ringle, C. M., \& Gudergan, S. P. (2018). Advanced issues in partial least squares structural equation modeling. Thousand Oaks, CA: Sage Publications.

Henseler, J., Ringle, C. M., \& Sarstedt, M. (2015). A new criterion for assessing discriminant validity in variancebased structural equation modeling. Journal of the Academy of Marketing Science, 43(1), 115-135. https://doi.org/10.1007/s11747-014-0403-8

Henseler, J., Ringle, C.M., \& Sinkovics, R. R. (2009). The use of partial least squares path modeling in international marketing. Advances in International Marketing, 20(1), 277-319. https://doi.org/10.1108/S1474-7979(2009)0000020014

Ho, H., \& Lu, R. (2015). Performance implications of marketing exploitation and exploration: Moderating role of supplier collaboration. Journal of Business Research, 68(5), 10261034. https://doi.org/10.1016/j.jbusres.2014.10.004

Jansen, J. J. P., Van Den Bosch, F. A. J, \& Volberda, H. W. (2006). Exploratory innovation, exploitative innovation, and performance: Effects of organizational antecedents and environmental moderators. Management Science, 52(11), 1661-1674. https://doi.org/10.1287/mnsc.1060.0576

Jansen, J. J. P., Vera, D., \& Crossan, M. (2009). Strategic leadership for exploration and exploitation: The moderating role of environmental dynamism. The Leadership Quarterly, 20(1), 5-18. https://doi.org/10.1016/j.leaqua.2008.11.008
Jarzabkowski, P., \& Kaplan, S. (2015). Strategy tools-in-use: A framework for understanding 'technologies of rationality' in practice. Strategic Management Journal, 36. https://doi.org/10.1002/smj.2270

Johnson, A. M., \& Lederer, A. L. (2013). IS strategy and IS contribution: CEO and CIO perspectives. Information Systems Management, 30(4), 306-318. https://doi.org/10.1080/10580530.2013.832962

Kamasak, R., Yozgat, U., \& Yavuz, M. (2017). Knowledge process capabilities and innovation: Testing the moderating effects of environmental dynamism and strategic flexibility. Knowledge Management Research and Practice, 15(3), 356368. https://doi.org/10.1057/s41275-017-0068-4

Kaplan, R. S., \& Norton, D. P. (2008). The execution premium: Linking strategy to operations for competitive advantage. Brighton, MA: Harvard Business School Press.

Kearns, G. S., \& Sabherwal, R. (2006). Strategic Alignment Between Business and Information Technology: A KnowledgeBased View of Behaviors, Outcome, and Consequences. Journal of Management Information Systems, 23(3), 129162. https://doi.org/10.2753/MIS0742-1222230306

King, W. R. (1978). Strategic Planning for Management Information Systems. MIS Quarterly, 2(1), 27-37. https://doi.org/10.2307/249104

Kohli, R., \& Grover, V. (2008). Business value of IT: An essay on expanding research directions to keep up with the times. Journal of the Association for Information Systems, 9(1), 2339. Retrieved from https://aisel.aisnet.org/jais/vol9/iss1/1/

Leal-Rodríguez, A. L., Ariza-Montes, A. J., \& Morales-fernández, E. (2017). Green innovation, indeed a cornerstone in linking market requests and business performance. Evidence from the Spanish automotive components industry. Technological Forecasting \& Social Change, 129, 185-193. https://doi.org/10.1016/j.techfore.2017.07.021

Leidner, D. E., Lo, J., \& Preston, D. S. (2011). An empirical investigation of the relationship of IS strategy with firm performance. Journal of Strategic Information Systems, 20(4), 419-437. https://doi.org/10.1016/j.jsis.2011.09.001

Lewin, A. Y., \& Volberda, H. W. (1999). Prolegomena on coevolution: A framework for research on strategy and new organizational forms. Organization Science, 10(5), 519-534. https://doi.org/10.1287/orsc.10.5.519

MacKenzie, S. B., \& Podsakoff, P. M. (2012). Common method bias in Marketing: Causes, mechanisms, and procedural remedies. Journal of Retailing, 88(4), 542-555. https://doi.org/10.1016/j.jretai.2012.08.001

Maletič, M., Maletič, D., \& Gomišček, B. (2016). The impact of sustainability exploration and sustainability exploitation practiceson theorganisational performance:Across-country comparison. Journal of Cleaner Production, 138 (Part 1), 158-169. https://doi.org/10.1016/j.jclepro.2016.02.132

Mao, H., Liu, S., \& Zhang, J. (2014). How the effects of IT and knowledge capability on organizational agility are contingent on environmental uncertainty and information intensity. Information Development, 31(4), 1-25. https://doi.org/10.1177/0266666913518059 
Marabelli, M., \& Galliers, R. D. (2017). A reflection on information systems strategizing: The role of power and everyday practices. Information Systems Journal, 27(3), 347-366. https://doi.org/10.1111/isj.12110

March, J. G. (1991). Exploration and exploitation in organizational learning. Organization Science, 2(1), 71-87. https://doi.org/10.1287/orsc.2.1.71

March, J. G. (1995). The Future, disposable organizations and the rigidities of imagination. Organization, 2(3-4), 427-440. https://doi.org/10.1177/135050849523009

Marôco, J. (2010). Análise de equaçōes estruturais: Fundamentos teóricos, software e aplicaçôes. [S.n]: Pêro Pinheiro.

Martinez-Simarro, D., Devece, C., \& Llopis-Albert, C. (2015). How information systems strategy moderates the relationship between business strategy and performance. Journal of Business Research, 68(7), 1592-1594. https://doi.org/10.1016/j.jbusres.2015.01.057

Matthews, L. M., Sarstedt, M., Hair, J. F., \& Ringle, C. M. (2016). Identifying and treating unobserved heterogeneity with FIMIX-PLS: Part II - A case study. European Business Review, 28(2), 208-224. https://doi.org/10.1108/EBR-09-2015-0095

Merali, Y., Papadopoulos, T., \& Nadkarni, T. (2012). Systems information systems strategy: Past, present, future? Journal of Strategic Information Systems, 21(2), 125-153. https://doi.org/10.1016/j.jsis.2012.04.002

Mikalef, P., \& Pateli, A. (2017). Information technologyenabled dynamic capabilities and their indirect effect on competitive performance: Findings from PLS-SEM and fsQCA. Journal of Business Research, 70, 1-16. https://doi.org/10.1016/j.jbusres.2016.09.004

Miles, R. E., Snow, C. C., Meyer, A. D., \& Coleman, H. J. (1978). Organizational strategy Structure and Process. Academy of Management Review, 3(3), 546-562. https://doi.org/10.5465/amr.1978.4305755

Mintzberg, H., Ahlstrand, B. W., \& Lampel, J. (2009). Strategy safari: The complete guide through the wilds of strategic management (2nd ed.). Upper Saddle River: Financial Times Prentice Hall.

Moeini, M., Rahrovani, Y., \& Chan, Y. E. (2019). A review of the practical relevance of IS strategy scholarly research. Journal of Strategic Information Systems, 28(2), 196-217. https://doi.org/10.1016/j.jsis.2018.12.003

Morgado, F. F. R., Meireles, J. F. F., Neves, C. M., Amaral, A. C. S., \& Ferreira, M. E. C. (2018). Scale development: Ten main limitations and recommendations to improve future research practices. Psychology: Research and Review, 30(1), 1-20. https://doi.org/10.1186/s41155-016-0057-1

Nan, N., \& Tanriverdi, H. (2017). Unifying The role of it in hyperturbulence and competitive advantage via a multilevel perspective of IS strategy. MIS Quaterly, 41(3), 937-958. https://doi.org/10.25300/MISQ/2017/41.3.12
Neirotti, P., \& Raguseo, E. (2017). On the contingent value of IT-based capabilities for the competitive advantage of SMEs: Mechanisms and empirical evidence. Information and Management, 54(2), 139-153. https://doi.org/10.1016/j.im.2016.05.004

Newkirk, H. E., \& Lederer, A. L. (2006). The effectiveness of strategic information systems planning under environmental uncertainty. Information \& Management, 43(4), 481-501. https://doi.org/10.1016/j.im.2005.12.001

Newkirk, H. E., \& Lederer, A. (2010). The impact of environmental dynamism on strategic information systems technical and personnel resources planning. International Journal of Information Technology and Management, 9(2), 203-223. https://doi.org/10.1504/IJITM.2010.030461

Panda, S., \& Rath, S. K. (2018). Information technology capability, knowledge management capability, and organizational agility: The role of environmental factors. Journal of Management \& Organization, 1-27. https://doi.org/10.1017/jmo.2018.9

Pavlou, P. A., \& El Sawy, O. A. (2006). From IT leveraging competence to competitive advantage in turbulent environments: The case of new product development. Information Systems Research, 17(3), 198-227. https://doi.org/10.1287/isre.1060.0094

Pavlou, P. A., \& El Sawy, O. A. (2010). The 'third hand': ITenabled competitive advantage in turbulence through improvisational capabilities. Information Systems Research, 21(3), 443-471. https://doi.org/10.1287/isre.1100.0280

Peng, D. X., \& Lai, F. (2012). Using partial least squares in operations management research: A practical guideline and summary of past research. Journal of Operations Management, 30(6), 467-480. https://doi.org/10.1016/j.jom.2012.06.002

Peppard, J., Galliers, R. D., \& Thorogood, A. (2014). Information systems strategy as practice: Micro strategy and strategizing for IS. Journal of Strategic Information Systems, 23(1), 1-10. https://doi.org/10.1016/j.jsis.2014.01.002

Philip, G. (2007). IS strategic planning for operational efficiency. Information Systems Management, 24(3), 247-264. https://doi.org/10.1080/10580530701404504

Popadiuk, S. (2012). Scale for classifying organizations as explorers, exploiters or ambidextrous. International Journal of Information Management, 32(1), 75-87. https://doi.org/10.1016/j.ijinfomgt.2011.07.001

Popadiuk, S., \& Bido, D. S. (2016). Exploration, exploitation , and organizational coordination mechanisms. $R A C$ Revista de Administraçâo Contemporânea, 20(2), 238-260. https://doi.org/10.1590/1982-7849rac2016150018

Popadiuk, S., Franklin, M. A., Vidal, P. G., Miguel, L. A. P., \& Prieto, V. C. (2010). Measuring Knowledge Exploitation and Exploration: An Empirical Application in a Technological Development Center in Brazil. Revista Espacios, 31(3), 36. Retrieved from https://www.researchgate. net/publication/280883281 Measuring knowledge exploitation and exploration An empirical application in a technological development center in Brazil 
Porter, M. E. (1990). Competitive advantage: Creating and sustaining superior performance. New York: Harvard Business Review.

Ray, G., Wu, D., \& Konana, P. (2009). Competitive environment and the relationship between IT and vertical integration. Information Systems Research, 20(4), 585-603. https://doi.org/10.1287/isre.1080.0202

Ringle, C. M., Bido, D. S., \& Silva, D. (2014). Structural equation modeling with the SmartPLS. Brazilian JournalofMarketing, 13(2), 56-73. https://doi.org/10.5585/remark.v13i2.2717

Ringle, C. M., Wende, S., \& Becker, J. (2015). SmartPLS 3. Bönningstedt: SmartPLS. Retrieved from http://www.smartpls.com

Rodríguez-Entrena,M.,Schuberth,F., \&Gelhard,C.(2018).Assessing statistical differences between parameters estimates in Partial Least Squares path modeling. Quality and Quantity, 52(1), 57-69. https://doi.org/10.1007/s11135-016-0400-8

Rouhani, S., Ashrafi, A., Ravasan, A. Z., \& Afshari, S. (2016). The impact model of business intelligence on decision support and organizational benefits. Journal of Enterprise Information Management, 29(1), 19-50. https://doi.org/10.1108/JEIM-12-2014-0126

Sabherwal, R., \& Chan, Y. E. (2001). Alignment between business and IS strategies: A study of prospectors, analyzers, and defenders. Information Systems Research. https://doi.org/10.1287/isre.12.1.11.9714

Scandelari, V., \& Cunha, J. (2013). Ambidestralidade e desempenho socioambiental de empresas do setor eletroeletrônico. Journal of Business Management, 53(2), 183-198. https://doi.org/10.1590/S0034-75902013000200006

Schilke, O. (2014). On the Contingent Value of Dynamic Capabilities for Competitive Advantage: the Nonlinear Moderating Effect of Environmental Dynamism. Academy of Management Journal, 51(2), 179-203. https://doi.org/10.1002/smj.02099

Schwarz, A., Rizzuto, T., Wolverton, C. C., \& Roldán, J. L. (2017). Examining the Impact and Detection of the "Urban Legend " of Common Method Bias. ACM SIGMIS Database: The DATABASE for Advances in Information Systems, 48(1), 93119. https://doi.org/10.1145/3051473.3051479

Shollo, A., \& Galliers, R. D. (2016). Towards an understanding of the role of business intelligence systems in organisational knowing. Information Systems Journal, 26(4), 339-367. https://doi.org/10.1111/isj.12071

Singh, S. K., Watson, H. J., \& Watson, R. T. (2002). EIS support for the strategic management process. Decision Support Systems, 33(1), 71-85. https://doi.org/10.1016/S0167-9236(01)00129-4

Sohn, M. H., You, T., Lee, S. L., \& Lee, H. (2003). Corporate strategies, environmental forces, and performance measures: A weighting decision support system using the k-nearest neighbor technique. Expert Systems with Applications, 25(3), 279-292. https://doi.org/10.1016/S0957-4174(03)00070-8
Song, M., Im, S., Van Der Bij, H., \& Song, L. Z. (2011). Does strategic planning enhance or impede innovation and firm performance? Journal of Product Innovation Management, 28(4), 503-520. https://doi.org/10.1111/j.1540-5885.2011.00822.x

Teubner, R. A. (2013). Theory, Practice, and Challenges for Future Research. Business \& Information Systems Engineerin, 5(4), 243-257. https://doi.org/10.1007/s12599-013-0279-z

Wang, Y., \& Hajli, N. (2017). Exploring the path to big data analytics success in healthcare. Journal of Business Research, 70, 287299. https://doi.org/10.1016/j.jbusres.2016.08.002

Whittington, R. (2014). Information Systems Strategy and Strategy-as-Practice: A joint agenda. Journal of Strategic Information Systems, 23(1), 87-91. https://doi.org/10.1016/j.jsis.2014.01.003

Wieland, A., Durach, C. F., Kembro, J., \& Treiblmaier, H. (2017). Statistical and judgmental criteria for scale purification. Supply Chain Management: An International Journal, 22(4), 321-328. https://doi.org/10.1108/SCM-07-2016-0230

Wilden, R., \& Gudergan, S. P. (2014). The impact of dynamic capabilities on operational marketing and technological capabilities: investigating the role of environmental turbulence.Journalof the AcademyofMarketingScience, 43(2), 181-199. https://doi.org/10.1007/s11747-014-0380-y

Wilhelm, H., Schlömer, M., \& Maurer, I. (2015). How dynamic capabilities affect the effectiveness and efficiency of operating routines under high and low levels of environmental dynamism. British Journal of Management, 26(2), 327345. https://doi.org/10.1111/1467-8551.12085

Wolf, C., \& Floyd, S. W. (2017). Strategic Planning Research: Toward a Theory-Driven Agenda. Journal of Management, 43(6), 1754-1788. https://doi.org/10.1177/0149206313478185

Xue, L., Ray, G., \& Sambamurthy, V. (2012). Efficiency or Innovation: How Do Industry Environments Moderate the Effects of Firms' It Asset Portfolios? MIS Quarterly, 36(2), 509-528. https://doi.org/10.2307/41703465

Yayla, A. A., \& Hu, Q. (2012). The impact of IT-business strategic alignment on firm performance in a developing country setting: exploring moderating roles of environmental uncertainty and strategic orientation. European Journal of Information Systems, 21(4), 373-387. https://doi.org/10.1057/ejis.2011.52

Yoshikuni, A. C., \& Albertin, L. A. (2017). IT-Enabled Dynamic Capability on Performance: an Empirical Study of BSC Model. Journal of Business Management, 57(maio-jun), 215-231. https://doi.org/10.1590/S0034-759020170303

Yoshikuni, A. C., \& Albertin, A. L. (2018). Effects of strategic information systems on competitive strategy and performance. International Journal of Productivity and Performance Management, 67(9), 2018-2045. https://doi.org/10.1108/IJPPM-07-2017-0166

Yoshikuni, A. C., Lucas, E. C., \& Albertin, A. L. (2019). Strategic Information Systems Enabling Strategy-as-Practice and Corporate Performance : Empirical Evidence from PLS-PM , FIMIX-PLS and fsQCA. International Business Research, 12(1), 131-147. https://doi.org/10.5539/ibr.v12n1p131 


\section{Autoria}

\section{Adilson Carlos Yoshikuni*}

Fundação Getulio Vargas, Escola de Administração de Empresas de São Paulo, Departamento de Tecnologia e Ciência de Dados Av. Nove de Julho, no 2029, Bela Vista, 01313-902, São Paulo, SP, Brasil.

E-mail: ayoshikuni@terra.com.br; adilson.yoshikuni@fgv.br

(1) https://orcid.org/0000-0003-4611-6933

\section{José Eduardo Ricciardi Favaretto}

Escola Superior de Propaganda e Marketing, Programa de Pósgraduação em Administração

Rua Dr. Álvaro Alvim, no 123, Vila Mariana, 04018-010, São Paulo, SP, Brasil.

E-mail: jose.favaretto@espm.br

(D) http://orcid.org/0000-0002-0143-0809

\section{Alberto Luiz Albertin}

Fundaçáo Getulio Vargas, Escola de Administraçáo de Empresas de São Paulo, Departamento de Tecnologia e Ciência de Dados Av. Nove de Julho, no 2029, Bela Vista, 01313-902, Sáo Paulo, SP, Brasil.

E-mail: albertin@fgv.br

(1) https://orcid.org/0000-0002-4851-0961

\section{Fernando de Souza Meirelles}

Fundação Getulio Vargas, Escola de Administraçáo de Empresas de São Paulo, Departamento de Tecnologia e Ciência de Dados

Av. Nove de Julho, no 2029, Bela Vista, 01313-902, São Paulo, SP, Brasil.

E-mail: fernando.meirelles@fgv.br

(1) https://orcid.org/0000-0002-0631-9800

* Autor Correspondente

\section{Financiamento}

Os autores relataram que não houve suporte financeiro para pesquisa deste artigo.

\section{Conflito de Interesses}

Os autores informaram que não há conflito de interesses.

\section{Verificação de Plágio}

A RAC mantém a prática de submeter todos os documentos aprovados para publicação à verificação de plágio, mediante o emprego de ferramentas específicas, e.g.: iThenticate.

\section{Direitos Autorais}

A RAC detém os direitos autorais deste conteúdo.

\section{Contribuições dos Autores}

$1^{2}$ autor: conceituação (igual); curadoria de dados (liderança); análise formal (liderança); investigação (liderança); metodologia (liderança); administração de projeto (liderança); recursos (igual); software (liderança); supervisão (liderança); validação (liderança); visualização (igual); escrita - rascunho original (liderança); escrita revisão e edição (igual).

$2^{\circ}$ autor: conceituação (igual); curadoria de dados (igual); análise formal (igual); investigação (igual); metodologia (igual); administração de projeto (igual); recursos (igual); software (igual); supervisão (igual); validação (igual); visualização (igual); escrita - rascunho original (igual); escrita - revisão e edição (igual).

$3^{\circ}$ autor: conceituação (suporte); curadoria de dados (suporte); análise formal (suporte); investigação (suporte); metodologia (suporte); supervisão (suporte); validação (suporte); escrita - rascunho original (suporte); escrita revisão e edição (igual).

$4^{\circ}$ autor: conceituação (suporte); análise formal (suporte); investigação (suporte); metodologia (suporte); administração de projeto (suporte); supervisão (suporte); validação (suporte); escrita - rascunho original (suporte); escrita - revisão e edição (suporte).

\section{Método de Revisão por Pares}

Este conteúdo foi avaliado utilizando o processo de revisão por pares duplo-cego (double-blind peer-review). A divulgação das informaçóes dos pareceristas constantes na primeira página e do Relatório de Revisão por Pares (Peer Review Report) é feita somente após a conclusão do processo avaliativo, e com o consentimento voluntário dos respectivos pareceristas e autores.

\section{Disponibilidade dos Dados}

Todos os dados e materiais foram disponibilizados publicamente por meio da plataforma Harvard Dataverse e podem ser acessados em:

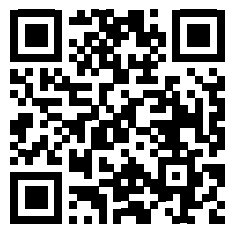

Adilson Carlos Yoshikuni; José Eduardo Ricciardi Favaretto; Alberto Luiz Albertin; Fernando de Souza Meirelles, 2020, "Replication Data for: How can strategy-as-practice enable innovation under the influence of environmental dynamism?", Harvard Dataverse, V1. https://doi.org/10.7910/DVN/ZXBMHD

A RAC incentiva o compartilhamento de dados mas, por observância a ditames éticos, não demanda a divulgação de qualquer meio de identificação de sujeitos de pesquisa, preservando a privacidade dos sujeitos de pesquisa. A prática de open data é viabilizar a reproducibilidade de resultados, e assegurar a irrestrita transparência dos resultados da pesquisa publicada, sem que seja demandada a identidade de sujeitos de pesquisa. 


\section{APÊNDICE A. ESCALA, ITENS E CARGA FATORIAL}

Os valores-p foram estimados por bootstrap ( $\mathrm{n}=256$ e 5.000 repetições).

Tabela A1. Escala, itens e carga fatorial do construto Strategic Information Systems.

\begin{tabular}{lcccc}
\hline Strategic information systems (SIS) & Carga fatorial & Erro padrão & Valor-t & Valor-p \\
\hline $\begin{array}{l}\text { SIS_1 - O SIS habilita a organização para disseminar e gerar consciência dos objetivos } \\
\text { estratégicos. }\end{array}$ & 0,844 & 0,024 & 35,647 & 0,000 \\
$\begin{array}{l}\text { SIS_2 - O SIS habilita a organização para mapear as oportunidades e ameaças do ambiente } \\
\text { externo. }\end{array}$ & 0,876 & 0,016 & 55,371 & 0,000 \\
SIS_3 - O SIS habilita a empresa para formular estratégias de negócios. & 0,918 & 0,010 & 90,839 & 0,000 \\
SIS_4 - O SIS habilita a empresa para executar as estratégias de negócio. & 0,842 & 0,018 & 47,805 & 0,000 \\
SIS_5-O SIS habilita a empresa para implantar e controlar os planos de açóes estratégicas. & 0,865 & 0,019 & 44,997 & 0,000 \\
\hline
\end{tabular}

Nota. Escala obtida de Yoshikuni e Albertin (2018).

Tabela A2. Escala, itens e carga fatorial do construto Inovação de exploration.

\begin{tabular}{|c|c|c|c|c|}
\hline Inovação de exploration & Carga fatorial & Erro padrão & Valor-t & Valor-p \\
\hline INEX_1 - A empresa aceita demandas que vão além dos produtos e serviços existentes. & (a) & & & \\
\hline INEX_2 - A empresa inventa novos produtos e serviços constantemente. & 0,834 & 0,024 & 35,072 & 0,000 \\
\hline INEX_3 - A empresa lança novos produtos e serviços para o mercado local. & 0,808 & 0,027 & 30,421 & 0,000 \\
\hline $\begin{array}{l}\text { INEX_4 - A empresa comercializa produtos e serviços que são completamente novos para } \\
\text { a unidade local. }\end{array}$ & 0,782 & 0,030 & 26,307 & 0,000 \\
\hline INEX_5 - A empresa com frequência identifica novas oportunidades em novos mercados. & 0,803 & 0,022 & 36,992 & 0,000 \\
\hline $\begin{array}{l}\text { INEX_6 - A empresa regularmente usa novos canais de distribuição para seus produtos } \\
\text { e serviços. }\end{array}$ & 0,762 & 0,029 & 25,882 & 0,000 \\
\hline
\end{tabular}

Nota. Escala obtida de Jansen et al. (2006). (a) Item eliminado por baixa carga fatorial: INEX_1 = 0,419.

Tabela A3. Escala, itens e carga fatorial do construto Inovaçáo de exploitation.

\begin{tabular}{lcccc}
\hline Inovação de exploitation & Carga fatorial & Erro padrão & Valor- $t$ & Valor-p \\
\hline INEP_1 - A empresa frequentemente aperfeiçoa os produtos e serviços existentes. & 0,822 & 0,026 & 31,420 & 0,000 \\
$\begin{array}{l}\text { INEP_2 - A empresa frequentemente implementa pequenas adaptaçôes nos produtos e } \\
\text { serviços existentes. }\end{array}$ & 0,725 & 0,043 & 17,089 & 0,000 \\
$\begin{array}{l}\text { INEP_3 - A empresa introduz melhorias apenas nos produtos e serviços existentes do } \\
\text { mercado local. }\end{array}$ & (a) & & & \\
$\begin{array}{l}\text { INEP_4 - A empresa possui foco na eficiência operacional na produção dos produtos e/ } \\
\text { ou entrega dos serviços. }\end{array}$ & 0,707 & 0,041 & 17,198 & 0,000 \\
$\begin{array}{l}\text { INEP_5 - A empresa possui foco no aumento da participação de mercado dos produtos } \\
\text { e serviços. }\end{array}$ & 0,773 & 0,036 & 21,515 & 0,000 \\
\begin{tabular}{l} 
INEP_6 - A empresa possui foco em expandir produtos e serviços para clientes existentes. \\
\hline
\end{tabular} & 0,775 & 0,036 & 21,541 & 0,000 \\
\hline
\end{tabular}

Nota. Escala obtida de Jansen et al. (2006). (a) Item eliminado por baixa carga fatorial: INEP_3 = 0,119.

Tabela A4. Escala, itens e carga fatorial do construto de dinamismo ambiental.

\begin{tabular}{|c|c|c|c|c|}
\hline Dinamismo ambiental & Carga fatorial & Erro padrão & Valor-t & Valor-p \\
\hline DINA_1 - Produtos e serviços em nosso setor tornam-se obsoletos rapidamente. & 0,525 & 0,099 & 5,294 & 0,000 \\
\hline DINA_2 - As tecnologias de produtos/serviços em nossa indústria mudam rapidamente. & 0,806 & 0,036 & 22,688 & 0,000 \\
\hline $\begin{array}{l}\text { DINA_3 - A taxa de inovação (voltada para a obtenção de vantagens competitivas e } \\
\text { alcance de objetivos de negócios) é alta em nossa indústria. }\end{array}$ & 0,875 & 0,022 & 39,834 & 0,000 \\
\hline
\end{tabular}

Nota. Escala obtida de Yayla \& Hu (2012). 